\title{
TIME INTEGRATION METHODS FOR REACTOR KINETICS
}

\author{
by
}

J. A. W. da Nóbrega, A. F. Henry

December, 1971

\begin{abstract}
Massachusetts Institute of Technology
Department of Nuclear Engineering

Cambridge, Massachusetts 02139
\end{abstract}

AEC Research and Development Report

Contract AT(11-1)-3052

U.S. Atomic Energy Commission 


\section{DISCLAIMER}

This report was prepared as an account of work sponsored by an agency of the United States Government. Neither the United States Government nor any agency Thereof, nor any of their employees, makes any warranty, express or implied, or assumes any legal liability or responsibility for the accuracy, completeness, or usefulness of any information, apparatus, product, or process disclosed, or represents that its use would not infringe privately owned rights. Reference herein to any specific commercial product, process, or service by trade name, trademark, manufacturer, or otherwise does not necessarily constitute or imply its endorsement, recommendation, or favoring by the United States Government or any agency thereof. The views and opinions of authors expressed herein do not necessarily state or reflect those of the United States Government or any agency thereof. 


\section{DISCLAIMER}

Portions of this document may be illegible in electronic image products. Images are produced from the best available original document. 


\title{
MASSACHUSETTS INSTTITUTE OF TECHNOLOGY \\ DEPARTMENT OF NUCLEAR ENGINEERING \\ Cambridge, Massachusetts 02139
}

\author{
TIME INTEGRATION METHODS \\ FOR REACTOR KINETICS
}

by
J. A. W. da Nobrega, A. F. Henry
December, 1971

NOTICE

This report was prepared as an account of work sponsored by the United States Government. Neither the-United States nor the United States Atomic Energy Commission, nor any of their employees, nor any of their contractors, subcontractors, or their employees, makes any warranty, express or implied, or assumes any legal liability. or responsibility for the accuracy, completeness or usefulness of any information, apparatus, product or process disclosed, or represents that its use would not infringe privately owned rights.

$$
\begin{aligned}
& \text { COO }-3052-1 \\
& \text { MITNE }-136
\end{aligned}
$$

AEC Research and Development Report

Contract AT $(11-1)-3052$

U. S. Atomic Energy Commission 
TIME INTEGRATION METHODS

FOR REACTOR KINETICS

by

José de Anchieta Wanderley da Nóbrega

Submitted to the Department of Nuclear Engineering on December 30, 1971, in partial fulfillment of the requirements for the degree of Doctor of Philosophy.

\section{ABSTRACT}

A technique based on the Padé approximations is applied to the solution of the point kinetics equations. The method consists of treating explicitly the roots of the inhour formula which would make the Pade approximations inaccurate. Also, an analytic method is developed which permits a fast inversion of polynomials of the point kinetics matrix and has direct applicability to the padé approximations.

Results are presented for several cases using various options of the method. It is concluded that the technique provides a fast and accurate computational method for the point kinetics equations.

Also, an implicit solution method for the time-dependent multigroup diffusion equations known as the "theta method" is studied. Both the usual method and a variation of it, derived from the precursor integrated equations, are considered. Several properties of both versions of the theta method are demonstrated.

An attempt is made to find better integration parameters (thetas) for the method, based on corresponding point kinetics calculations. Calculations are done for several test cases, leading to the conclusion that the improvements obtained are of limited value.

Thesis Supervisor: Allan F Henry Title: Professor of Nuclear Engineering 
TABLE OF CONTENTS

Page

ABSTRACT

LIST OF TABLES

LIST OF FIGURES

ACKNOWLEDGMENTS

BIOGRAPHICAL NOTE

Chapter 1. INTRODUCTION

Chapter 2. SOLUTION OF THE POINT KINETICS EQUATIONS

2.I The Point Kinetics Equations

2.2 An Approximation for exp (hA)

2.3 The Padé Approximations and Related Inversions 20

2.3.1 The Padé $(1,1)$ Approximation

2.3.2 The Padé $(2,0)$ Approximation.

2.4 Analysis of the Assumption of Constant Parameters
Chapter 3. SOLUTION OF THE SPACE-DEPENDENT KINETICS EQUATIONS

3.1 The Space-Dependent Kinetics Equations 30

3.2 Spatial Discretization 32

3.3 Survey of Solution Methods 35

3.4. Derivation of the Theta-Differenced Equations 37

3.5 Determination of the Parameters Theta 43

3.6 Properties of the Solution Method 46

3.6.1 Consistency 
Page

3.6 .2 stability

3.6.3 Steady-State Behavior

3.6.4 Asymptotic Behavior

3.6.5 Solvability

Chapter 4. NUMERICAL RESULTS

4.1 Results for Point Kinetics 61

4.2 Analysis of point kinetics Results 71

4.3 Results for Space-Dependent Kinetics 73

4.4 Analysis of Space Kinetics Results 89

4.5 Additional Results for Space-Dependent Kinetics

Chapter 5. CONCLUSIONS AND RECOMMENDATIONS $\cdots 97$

5.1 Conclusions for Point kinetics $\quad 97$

5.2 Conclusions for Space-Dependent Kinetics 99

5.3 Recommendations for Further Research $\quad 101$

REFERENCES $\quad . \quad 104$

Appendix A. CALCULATION OF THE EIGENVALUES OF LARGE
MAGNTUDE

Appendix B. CALCULATION OF THE PARAMETERS THETA 110

Appendix C. PROOF OF EQUIVALENCE OF NORMS 113

Appendix D. INPUT CARDS FOR COMPUTER PROGRAMS 115

APpendix E. LISTINGS OF COMPUTER PROGRAMS 121

(Only in first six copies) 
No.

Page

I Parameters for Two Typical Reactors

II Percent Errors and Exact $n(t)$ for Case 1 .

III Percent Errors and Exact $n(t)$ for Case 2

IV Percent Errors and Exact $n(t)$ for Case 3

V Percent Errors and Exact $n(t)$ for Case $4 \ldots 9$

VI Percent Errors and Exact $n(t)$ for Case $5 \quad 70$

VII Parameters for a Thermal Reactor $\quad 74$

VIII Parameters for a Fast Reactor 75

IX Flux Values for Case A 78

$\mathrm{X}$ Flux Values for Case $\mathrm{B}$

XI Flux Values for Case C 83

XII Flux Values for Case D : 85

XIII Flux Values for Case $E$. 88

XIV Percent Errors for a Delayed Critical Ramp 93

XV Ratios of Relative Error and Time Step Size 93 


\section{IIST OF FIGURES}

No.

Page

1

Geometry for a Thermal Reactor

76

2

Geometry for a Fast Reactor 


\section{ACKNOWLEDGEMENTS}

The author wishes to express his deep appreciation to Professor Allan F. Henry for his valuable guidance and suggestions during the course of this work.

Thanks are also extended to Professor Kent. F. Hansen for some helpful comments and discussions.

The author's wife, Else, provided encouragement and help during this work, particularly in preparing the first draft. Her effort is very much appreciated.

All computations were done at the M.I.T. Information Processing Center. This work was performed under the auspices of the U. S. Atomic Energy Commission. 


\section{BIOGRAPHICAI NOTE}

José de Anchieta Wanderley da Nobrega was born on April 2, 1941, in Campina Grande, Parafba, Brazil. He received his primary and secondary education in Campina Grande; and was graduated from the Colegio Estadual de Campina Grande in December, 1958.

He enrolled at the Escola Nacional de Engenharia of the Universidade do Brasil (now Universidade Federal do Rio de Janeiro) in March, 1959. He received his degree in Electrical Engineering (S.B. equivalent) in December, 1963.

After graduation, he participated of a two-month technical tour through several European countries. He worked at the Instituto de Engenharia Nuclear, Rio de Janeiro, Brazil until August, 1967. During the first half of 1967 he took, concurrently, a few graduate courses in Electrical Engineering at the Universidade Federal do Rio de Janeiro.

In september, 1967, he entered the Massachusetts Institute of Technology with an I.A.E.A. fellowship, having received a Master of Science degree in Nuclear Engineering in september, 1968. He continued his graduate studies at M.I.T.

Mr. da Nobrega is married to the former Else Joana Schumacher of Petropolis, Rio de Janeiro, Brazil and they have two children, Ricardo and Alessandra. 
Chapter 1

INTRODUCTION

In a nuclear reactor, several kinds of time dependent calculations must be done to assure an economical design and a safe operation. These calculations span time scales several orders of magnitude apart and can be grouped in some general categories.

First, there are calculations with a time scale of weeks or months. These include fuel depletion, buildup of long lived fission products, breeding of new fissile material and poison management, if any. Second, there are calculations with a time scale of hours, namely, overall xenon concentration changes and xenon spatial oscillations. Finally, a third class of calculations is concerned with short-term transients, with time scales of minutes to fractions of a second. The latter involves variations in power, temperature, pressure, and coolant flow and some of their consequences, like fuel expansion, moderator and coolant concentration changes, coolant voiding, Doppler effects, and control rod motion. Of course, some of the aforementioned problems are important or existent for only certain types of reactors. This thesis is concerned with the solution of problems in the third class.

To treat the short-term transients, the simplest approach is provided by the point kinetics model, described by the point kinetics equations, in which only the time depen- 
dence of the total neutron population for some related quantity) is followed. This model is useful to describe the effects of perturbations which involve small changes in the flux shape and is also widely used for stability analysis in reactor control theory. In addition, the point kinetics equations are part of the solution of some space-dependent models, and will in fact be also used in this thesis in such a role.

It is therefore of some interest to have an efficient computational method for the solution of the point kinetics equations. In Chap. 2 we develop a new numerical method for the solution of these equation which is fast and accurate, when compared to some previous methods, particularly for perturbations resulting from a fixed insertion of reactivity.

The point kinetics model is inadequate, however, for certain types of short-term transient analyses especially in large loosely-coupled cores. These involve mainly cases in which spatial changes in the flux shape are considerable so that the underlying assumption of the point kinetics model (that only the amplitude and not the shape of the flux changes during a transient) is made invalid. For this reason, the more accurate space-dependent kinetics models are being used increasingly. So far, spatial kinetics has been based almost entirely on the multigroup diffusion model, because of its relative computational simplicity.

In Chapter 3 we study a time integration technique for 
the time dependent multigroup diffusion equations based on the approach used in the "theta method". $(1,2)$ This is an implicit method which permits comparatively large time steps to be taken, although at the cost of requiring a considerable amount of computation time per step, because of its implicit nature. In this work we attempt to use the point kinetics equations in order to find better integration parameters (thetas) for the space-time equations. The ultimate goal is to improve the accuracy of a given space-time calculation or to increase the time step size demanded for a given accuracy. A variation of the theta method, which uses the precursor integrated equations, is also considered in Chap. 3. Several properties of the two versions are demonstrated in that chapter.

In both the point and space-dependent kinetics equations to be considered, the time dependent behavior of the reactor parameters is assumed given, whether such behavior is externally induced or is due to feedback effects. The latter are supposed to have been calculated by some approximate method not explicitly discussed in this work.

In Chapter 4 , results are presented for some point kinetics problems, using the method of Chap. 2, as well as for some space-dependent problems, using the techniques considered in chap. 3.

Finally, chap. 5 presents the conclusions reached in this work and offers some recommendations for further research. The technique of chap. 2 is found to be, in fact, a 
fast and accurate computational method for point kinetics. Also, it is concluded that only limited improvements are obtained by the use of point kinetics as a means to find better time integration parameters for the space-dependent kinetics equations. 


\author{
Chapter 2 \\ SOLUTION OF THE POINT KINETICS EQUATIONS
}

\title{
2.1 The Point Kinetics Equations
}

For various reasons, the point kinetics equations are still an important set of equations in this era of spacedependent kinetics. First, they can be derived formally from a transport theory formulation ${ }^{(3)}$ and will lead to quite accurate results if a good approximation for the flux shape is available. Thus they are useful for describing the effects of small perturbations or in rough preliminary reactor calculations, particularly for tightly coupled cores. Secondly, they are widely applied in the control theory aspects of reactor analysis, where it is difficult to perform a study using a full space-time description of the neutronics. $(4,5)$ In addition, some of the more elaborate spacetime methods rely on the solution of the point kinetics equations, namely the family of quasi-static methods ${ }^{(6)}$ and some synthesis schemes. (7) In some of these methods a generalized point kinetics formulation results in which the elements of the ordinary point kinetics equations are replaced by matrices having a similar, but more generalized, physical meaning. $(7,8)$

We feel therefore justified in developing an efficient solution method for the point kinetics equations, even recognizing that they do not offer an insurmountable barrier for present-day computers, whatever the solution technique 
adopted.

The preferred form of the point kinetics equations is written in terms of the neutron generation time. (9) They are:

$$
\begin{aligned}
& \frac{d n}{d t}=\frac{\rho-\beta}{\Lambda} n+\sum_{i=1}^{I} \lambda_{i} c_{i}+f \\
& \frac{d c_{i}}{d t}=\frac{\beta_{i}}{\Lambda} n-\lambda_{i} c_{i} \quad(i=1,2, \ldots, I)
\end{aligned}
$$

where $n$ and the $c_{i}$ are weighted integrals of the neutron and $i^{\text {th }}$ precursor concentrations; $f$ is a source term; $\rho$ is the reactivity; $\beta_{i}$ is the effective delayed neutron fraction for group $i_{i} \lambda_{i}$ is the decay constant for precursor $i ; \Lambda$ is the neutron generation time; and $I$ is the total number of delayed neutron groups. The quantities $n, c_{i}$ ! $f$ and $\rho$ are, in general, functions of the time $t$ and $\beta_{i}, \lambda_{i}$ and $\Lambda$ are assumed constant. In addition, $\rho$ may be a function of $n$ in feedback problems.

The above equations can be written in matrix form as

$$
\frac{d \underline{\Psi}}{d t}=A \underline{\Psi}+\underline{\underline{f}}
$$

where $\underline{\Psi}=\operatorname{col}\left[\begin{array}{llllll}n & c_{1} & c_{2} & \ldots & c_{I}\end{array}\right], \underline{f}=\operatorname{col}\left[\begin{array}{lllll}f & 0 & 0 & \ldots & 0\end{array}\right]$, and

$$
A=\left[\begin{array}{ccccc}
\alpha & \lambda_{1} & \lambda_{2} & \cdots & \lambda_{I} \\
\mu_{I} & -\lambda_{1} & 0 & \cdots & 0 \\
\mu_{2} & 0 & -\lambda_{2} & \cdots & 0 \\
\vdots & \vdots & \vdots & \ddots & \vdots \\
\mu_{I} & 0 & 0 & \cdots & -\lambda_{I}
\end{array}\right],
$$


with $\alpha=(\rho-\beta) / \Lambda,\left(\beta=\sum \beta_{i}\right)$ and $\mu_{i}=\beta_{i} / \Lambda$. A is usually called the point kinetics matrix.

When $\rho$ and $f$ vary with time, Eq. (1) is usually solved in a series of time steps, the assumption being made that $p$ and $f$ are constant and equal to their average values during the time step under consideration. Later we shall analyze the implications of this assumption. For constant A the formal solution of Eq. (1) is given by

$$
\underline{\Psi}_{1}=e^{h A} \underline{\Psi}_{0}+A^{-1}\left[e^{h A}-I\right] \underline{f}^{\prime}
$$

where $h=t_{1}-t_{0}$ and $\underline{\Psi}_{0}, \underline{\Psi}_{1}$ are the values of the vector $\underline{\Psi}$ at the beginning and end of the time step, respectively. Note that the matrix function multiplying $\underline{f}$ is always well defined, even if $A$ is singular.

Because this system of equation is a relatively simple one, its solution can be done in practice by "brute-force" methods, that is, calculating all the eigenvalues of the matrix $A$ and performing straightforward computations. However, this is an expensive scheme, when the reactivity varies with time, since the calculation of the eigenvalues amounts to solving and $(I+1)^{\text {th }}$ order algebraic equation (the inhour formula) for all its roots at every time step.

Thus a wealth of methods have been devised to solve Eq.

(1) in a more economical way. Among them we have: i) methods based essentially on Taylor series expansions; $(10,11)$ ii) methods based on convolution integrals using numerical 
integration; $(12,13)$ iii) methods based on integral equation formulations and approximation of the integrand; $(14-17)$ iv) methods based on some approximation of matrix exponentials; $(18,19)$ v) methods based on extrapolation of low order approximations; ${ }^{(20)}$ vi) methods using spline functions. of course there are many other methods and we do not intend the above list to be exhaustive.

In what follows, we propose to apply yet another method which is expected to be very fast and accurate and which has the ability to reproduce all the features of transients, including the prompt jump, which is not very well represented in some of the other methods.

our method is based on an approximate expression for $e^{\text {hA }}$, which was suggested by an earlier scheme called the "purification method".(21) Also important in the present work is a procedure to invert polynomials of the point kinetics matrix $A$, which might be considered a good method of solution for Eq. (1) in its own right, as we shall see later.

\subsection{An Approximation for $\exp (\mathrm{hA})$}

As seen in the last section there is a need for a simple and accurate computational method to evaluate $\exp (\mathrm{hA})$. In this section we derive a method which we believe to possess these characteristics. The essential idea will be to replace $\exp (\mathrm{hA})$ by an approximation $f(h A)$ plus a correction term to account properly for those eigenvalues of $A$ for 
which the approximation is inaccurate.

Let the matrix $A$ have eigenvalues $\omega_{i}$ and eigenvectors

$\underline{u}_{i}$. Also let $A^{T}$ have eigenvectors $\underline{v}_{i}$, corresponding to eigenvalues $\omega_{i}$ which are the same as those of $A$. The eigenvalues $\omega_{i}$ are the roots of the inhour formula

$$
\rho=\omega \Lambda+\omega \sum_{i=1}^{I} \frac{\beta_{i}}{\lambda_{i}+\omega}
$$

and can be ordered as

$$
\omega_{I}<-\lambda_{I}<\omega_{I-1}<-\lambda_{I-1}<\ldots<\omega_{1}<-\lambda_{1}<\omega_{0} .
$$

We now state the following

THEOREM: If $A \underline{u}_{i}=\omega_{i} \underline{u}_{i}$ and $A^{T} \underline{v}_{i}=\omega_{i} \underline{v}_{i}$ with $\underline{u}_{i}^{T} \underline{v}_{i}=1$, that is, normalized to unity, the following expression holds for any $f(A)$ for which $f\left(\omega_{i}\right)$ is bounded for all $i$ :

$$
e^{A}=f(A)+\sum_{i=0}^{I}\left[e^{\omega_{i}}-f\left(\omega_{i}\right)\right] \underline{u}_{i} \underline{v}_{i}^{T} .
$$

Proof: Since $e^{A} \underline{u}_{k}=e^{\omega_{k}} \underline{u}_{k}$ for all $\underline{u}_{k}$ we have only to show that this same relationship holds for the right hand side of Eq. (5), since two matrices with the same eigenvalues and eigenvectors are identical.

Thus we write

$$
e^{A} \underline{u}_{k}=f(A) \underline{u}_{k}+\sum_{i=0}^{I}\left[e^{\omega_{i}}-f\left(\omega_{i}\right)\right] \underline{u}_{i} \underline{v}_{i}^{T} \underline{u}_{k} .
$$

But $\underline{v}_{i}^{T} \underline{u}_{k}=\delta_{i k}$, since the eigenvalues of $A$ and $A^{T}$. form a biorthonormal set when properly normalized. (22) As a re- 
sult,

$$
\begin{aligned}
e^{A} \underline{u}_{k} & =f\left(\omega_{k}\right) \underline{u}_{k}+\sum_{i=0}^{I}\left[e^{\omega_{i}}-f\left(\omega_{i}\right)\right] \underline{u}_{i} \delta_{i k} \\
& =f\left(\omega_{k}\right) \underline{u}_{k}+\left[e^{\omega_{k}}-f\left(\omega_{k}\right)\right] \underline{u}_{k}=e^{\omega_{k}} \underline{u}_{k} \quad \text { Q.E.D. }
\end{aligned}
$$

To suit our particular needs we introduce the factor $h$. Equation (5) then becomes

$$
e^{h A}=f(h A)+\sum_{i=0}^{I}\left[e^{h \omega_{i}}-f\left(h \omega_{i}\right)\right] \underline{u}_{i} \underline{v}_{i}^{T}
$$

with $\underline{u}_{i}$ and. $\underline{v}_{i}$ unchanged, since $(h A) \underline{u}_{i}=\left(h \omega_{i}\right) \underline{u}_{i}$ and analogously for $\underline{v}_{i}$.

Equation (6) is so far a mere mathematical manipulation. It has, however, a form which will permit us to approximate $e^{h A}$ in an economical fashion. We only have to note that, if $f\left(h \omega_{i}\right)$ is a good approximation for $e^{h \omega_{i}}$, we are justified in dropping the $i^{\text {th }}$ term from the summation. It will have a very small coefficient, namely

$$
\left[e^{h \omega_{i}}-f\left(h \omega_{i}\right)\right] \ll 1,
$$

since $e^{h \omega_{i}} \simeq f\left(h \omega_{i}\right)$.

Thus, to a high degree of accuracy, we have.

$$
e^{h A} \simeq g(h A) \equiv f(h A)+\sum_{k}^{1}\left[e^{h \omega_{k}}-f\left(h \omega_{k}\right)\right] \underline{u}_{k} \underline{v}_{k}^{T} ;
$$

where the sum $\Sigma^{\prime}$ is over only those $k$ for which Eq. (7) does not hold.

Another way to see the small size of the errors invol- 
ved is to calculate the Euclidean norm of the difference between the exact exponential and the approximation $g(h A)$. From Eqs. (6) and (8), we have

$$
\left\|e^{h A}-g(h A)\right\|=\left\|\sum_{i}^{n}\left[e^{h \omega_{i}}-f\left(h \omega_{i}\right)\right] \underline{u}_{i} \underline{v}_{i}^{T}\right\|,
$$

where $\Sigma "$ runs over all $i$ for which Eq. (7) holds, that is, those excluded from the summation $\Sigma^{\prime}$ in Eq. (8). Using the properties of norms ${ }^{(23)}$ we have:

$$
\left.\left\|e^{h A}-g(h A)\right\| \leq \sum_{i}^{\prime \prime} \mid e^{h \omega} i_{-f\left(h \omega_{i}\right.}\right) \mid \cdot\left\|\underline{u}_{i}\right\| \cdot\left\|\underline{v}_{i}\right\|
$$

Since $\underline{u}_{i}^{\mathrm{T}} \underline{\mathrm{v}}_{i}=1,\left\|\underline{u}_{i}\right\| \cdot\left\|\underline{\mathrm{v}}_{i}\right\| \geq 1$ but is expected not to be very large. From Eq. (7) we can then conclude that the norm in question is indeed small and that, accordingly, $g(\mathrm{hA})$ is a good approximation for the exponential:

In order to implement the approximation (8) we need an expression for the eigenvectors $\underline{u}_{k}$ and $\underline{v}_{k}$ of the matrix $A$ and its transpose. These are easily calculated from their defining equations. They are:

$$
\begin{aligned}
& \underline{u}_{k}=\operatorname{col}\left[1 \frac{\mu_{1}}{\lambda_{1}+\omega_{k}} \frac{\mu_{2}}{\lambda_{2}+\omega_{k}} \cdots \frac{\mu_{I}}{\lambda_{I}+\omega_{k}}\right] \\
& \underline{v}_{k}=\nu_{k} \operatorname{col}\left[1 \frac{\lambda_{1}}{\lambda_{1}+\omega_{k}} \frac{\lambda_{2}}{\lambda_{2}+\omega_{k}} \cdots \frac{\lambda_{I}}{\lambda_{I}+\omega_{k}}\right]
\end{aligned}
$$

where $v_{k}$ is a normalization factor given by

$$
v_{k}=\left(1+\sum_{i=1}^{I} \frac{\mu_{i} \lambda_{i}}{\left(\lambda_{i}+\omega_{k}\right)^{2}}\right)^{-1}<1
$$


The present method is thus seen to require calculation of only those $w_{k}$ which are included in $\Sigma^{\prime}$ of $\mathrm{Eq} \cdot(8)$. In practice, only $\omega_{0}$ and $\omega_{I}$ are ever computed. Appendix $A$ describes a procedure to find $\omega_{0}$ and $\omega_{I}$ from the inhour formula, Eq. (3), using the Newton-Raphson method.

\subsection{The Pade Approximations and Related Inversions}

In order to use the method derived in the previous section, we need to find an approximation $f(x)$ for $e^{x}$ such that Eq. (7) holds for most eigenvalues $\omega_{i}$ ' so that only one, or at most two terms have to be included in $\Sigma^{\prime}$ of Eq. (8). Another requirement for $f(x)$ is that the computation of $f(h A)$ can be done without too much effort.

A particular class of approximations that fulfills these conditions are the Pade rational approximations. These approximations are known to be consistent and unconditionally stable when the numerator is a polynomial of the same degree as the denominator or smaller. (24) For any of these approximations for which the degree of the polynomial of the denominator is larger than unity we will have a full square matrix of order $(I+1)$ to invert, which is a task one normally tries to avoid, particularly for the case of varying reactivity when such inversion needs to be done at every time step.

We have, however, developed a method in which, by going temporarily to the complex plane, we obtain simple analytic expressions for such inverses. As a result, essentially the 
same number of arithmetic operations suffice to evaluate the product of the inverse of a polynomial of the matrix $A$ and $a$ vector as are required to compute the product of the polynomial itself and a vector. This fact makes the computational effort involved in using implicit methods of any order equal to that used for explicit methods of the same order (Taylor series expansions). However, instabilities associated with the latter are avoided.

The method is based on an expression for the inverse of [I- $\varepsilon A]$ where $\varepsilon$ is any scalar such that $[I-\varepsilon A]$ is non-singular. This expression is

$$
:[I-\varepsilon A]^{-1}=\gamma \underline{a} \underline{b}^{T}+D,
$$

where

$$
\begin{aligned}
& \gamma=\left(1-\varepsilon \frac{\rho}{\Lambda}+\sum_{i=1}^{I} \frac{\varepsilon \mu_{i}}{1+\varepsilon \lambda_{i}}\right)^{-1} \\
& \underline{a}=\operatorname{col}\left[1 \frac{\varepsilon \mu_{1}}{1+\varepsilon \lambda_{1}} \frac{\varepsilon \mu_{2}}{1+\varepsilon \lambda_{2}} \cdots \frac{\varepsilon \mu_{I}}{1+\varepsilon \lambda_{I}}\right] \\
& \underline{b}=\operatorname{col}\left[1 \frac{\varepsilon \lambda_{1}}{1+\varepsilon \lambda_{1}} \frac{\varepsilon \lambda_{2}}{1+\varepsilon \lambda_{2}} \cdots \frac{\varepsilon \lambda_{I}}{1+\varepsilon \lambda_{I}}\right] \\
& D=\operatorname{diag}\left[0 \frac{1}{1+\varepsilon \lambda_{1}} \frac{1}{1+\varepsilon \lambda_{2}} \cdots \frac{1}{1+\varepsilon \lambda_{I}}\right]
\end{aligned}
$$

The validity of Eq. (9) can be verified directly by multiplication by $[I-\varepsilon A]$.

Notice that only $\gamma$, a scalar, depends on the reactivity. Also it is worth noting the computational advantages of multiplying an $\mathrm{N}$-dyad by a vector, as compared to a 
general $\mathrm{N} \times \mathrm{N}$ matrix. The former involves only $3 \mathrm{~N}$ arithmetic operations as compared to $2 \mathrm{~N}^{2}$ in the latter. We further observe that, for this particular matrix $A$, essentially the same number of operations are required to effect the product of either $[I-\varepsilon A]^{-1}$ or $[I+\varepsilon A]$ and some vector.

The expression (9) is of no great advantage by itself since we can always solve directly the system of equations implied by the inverse shown, spending essentially the same computational effort, in this case. The utility of the analytical inversion is evident, however, when one tries to invert a general polynomial of the matrix $A$ which can be expressed as a product of factors having the form $[I-\varepsilon A]$. To see this utility in more detail, consider the matrix polynomial

$$
P_{N}(A)=\sum_{n=0}^{N} C_{n} A^{n}
$$

with $\left\{c_{n}\right\}$ being real numbers and $c_{0}=1$.

We factor $P_{N}(A)$ as

$$
P_{N}(A)=\prod_{n=1}^{N}\left[I-\varepsilon_{n} A\right],
$$

where $\left\{\varepsilon_{n}\right\}$ are, in general, complex numbers. Then,

$$
\left[P_{N}(A)\right]^{-1}=\prod_{n=1}^{N} \cdot\left[I-\varepsilon_{n} A\right]^{-1}
$$

of course, the $\left\{\varepsilon_{n}\right\}$ will either be real numbers or form complex conjugate pairs. For $\varepsilon_{\mathrm{n}}$ real we use Eq. (9) to do the inversion or directly solve the pertinent system of equations. For a complex conjugate pair we consider the 
pair of factors

$$
[I-\varepsilon A]^{-1}\left[I-\bar{\varepsilon}_{A}\right]^{-1}=I-2 \operatorname{Re}(\varepsilon) A+|\varepsilon|^{2} A^{2},
$$

which is a real matrix and thus has a real inverse. By using Eq. (9) with complex $\varepsilon$ for both factors and combining the complex elements of the resulting expression we will get: a sum of two real dyads and one real diagonal matrix as the desired inverse. Almost all of the elements involved can be precomputed since only the reactivity changes with time and it appears in a simple fashion in the coefficients of the dyads as we shall see later in a specific example.

For the Padé approximations Eq. (7) will be satisfied, in general, whenever $\left|h \omega_{i}\right| \ll 1$ and therefore we can use this as a preliminary criterion to decide which terms should be included in Eq. (8). From Eq. (4) we see that, with the exception of $\omega_{I}$ and, possibly, $\omega_{0}$, all the eigenvalues of the matrix A have magnitudes smaller than $\lambda_{I}$. Therefore, the condition $h \lambda_{I} \ll 1$ assures that Eq. (7) will be satisfied for most eigenvalues $\omega_{i}$. For practical values of $\lambda_{I^{\prime}}$ this allows that relatively large values of $h$ be taken with good accuracy.

As an example, we consider two of the Padé approximations, namely the Crank-Nicholson and the second order implicit approximations.

\subsubsection{The Pade $(1,1)$ Approximation}

This approximation, commonly known as the Crank-Nichol- 
son approximation, is given by

$$
f_{1}(h A)=\left[I-\frac{1}{2} h A\right]^{-1}\left[I+\frac{1}{2} h A\right]
$$

and involves no particular difficulty, except that one must always choose $h$ so that $h \omega_{i} \neq 2$ to keep $f\left(h \omega_{i}\right)$ bounded. Since only the eigenvalue $\omega_{0}$ of A can be positive we simply must have $h \omega_{0} \neq 2$. For small $h$, the error of this aproximation is given by

$$
e^{h A}-f_{I}(h A)=-\frac{1}{12} h^{3} A^{3}+0\left(h^{4}\right)
$$

Since all eigenvalues of $A$ have small magnitudes except possibly $\omega_{0}$ and $\omega_{6}$ these are the only ones we will ever need to calculate explicitly for use in Eq. (8), if we do not want to take $h$ very small. Also, we have that $\left|e^{h \omega_{i}}-f_{l}\left(h \omega_{i}\right)\right|$ $<0.035$ or 0.14 for negative $h \omega_{i}$ down to -1 or -2 , respectively. These figures are low enough to require the inclusion of the ${ }^{\omega_{I}}$ term in Eq. (8) only when $h \omega_{I}<-1$ or, in many cases, when $h \omega_{I}<-2$. Such conditions are clearly much less stringent than the general condition: $\left|h \omega_{I}\right| \ll 1$. The above considerations, coupled with the fact that for most practical cases only one of $\omega_{0}$ and $\omega_{I}$ is of large magnitude, indicate that, in many problems, satisfactory results will be obtained by treating explicitly only one term in Eq. (8).

\subsubsection{The Pade $(2,0)$ Approximation}

This approximation, which we call the second order 
implicit approximation, is given by

$$
f_{2}(h A)=\left[I-h A+\frac{1}{2} h^{2} A^{2}\right]^{-1},
$$

and $f_{2}\left(h \omega_{i}\right) \neq 0$ for any real value of $h \omega_{i}$. For small $h$ the error of this approximation is given by

$$
e^{h A}-f_{2}(h A)=\frac{1}{6} h^{3} A^{3}+o\left(h^{4}\right) .
$$

We will show here an explicit application of the method which we developed, to find the inverse in $f_{2}(h A)$. We factor $f_{2}(h A)$ as

$$
f_{2}(h A)=[I-\varepsilon A]^{-1}[I-\overline{\varepsilon A}]^{-1},
$$

where $\varepsilon=h(1+i) / 2$ with $i=\sqrt{-1}$.

Using Eq. (9) for these values of $\varepsilon$ and carrying out

some involved but straightforward algebra we eliminate all imaginary numbers in the final expression which is given by

$$
f_{2}(h A)=\gamma_{1}\left(\underline{a}_{1} \underline{b}_{2}^{T}+\underline{a}_{2} \underline{b}_{1}^{T}\right)+\gamma_{2}\left(\underline{a}_{1} \underline{b}_{1}^{T}-\underline{a}_{2} \underline{b}_{2}^{T}\right)+D^{\prime}
$$

where

$$
\begin{array}{ll}
\gamma_{1}=2 r /\left(r^{2}+s^{2}\right) ; & r_{2}=2 s /\left(r^{2}+s^{2}\right) ; \\
r=1-\frac{h \rho}{\Lambda}+h \sum_{i=1}^{I} \mu_{i} p_{i}\left(2+h \lambda_{i}\right) ; & \\
s=1+h^{2} \sum_{i=1}^{I} \mu_{i} p_{i} \lambda_{i} ; & p_{i}=\left[1+\left(1+h \lambda_{i}\right)^{2}\right]^{-1} ;
\end{array}
$$




$$
\begin{aligned}
& \underline{a}_{1}=\left[\begin{array}{c}
1 \\
h \mu_{1} p_{1}\left(1+h \lambda_{1}\right) \\
h \mu_{2} p_{2}\left(1+h \lambda_{2}\right) \\
\ldots \\
h \mu_{I} p_{I}\left(1+h \lambda_{I}\right)
\end{array}\right] ; \quad \underline{a}_{2}=\left[\begin{array}{c}
0 \\
h \mu_{I} p_{1} \\
h{ }_{2} p_{2} \\
\ldots \\
h \mu_{I} p_{I}
\end{array}\right] ; \\
& \underline{b}_{1}=\left[\begin{array}{c}
h \lambda_{2} p_{2}\left(1+h \lambda_{2}\right) \\
\cdot \ldots \\
h \lambda_{1} p_{1}\left(1+h \lambda_{1}\right) \\
h \lambda_{I} p_{I}\left(1+h \lambda_{I}\right)
\end{array}\right] ; \quad \underline{b}_{2}=\left[\begin{array}{c}
h \lambda_{1} p_{1} \\
h \lambda_{2} p_{2} \\
\ldots \\
h \lambda_{I} p_{I}
\end{array}\right] ; \\
& \vdots
\end{aligned}
$$

We note that only the scalars $r, \gamma_{1}$ and $\gamma_{2}$ depend on the reactivity and therefore almost everything can be precomputed. Also, although we have displayed four dyads in the expression for $\mathrm{f}_{2}(\mathrm{hA})$ we could combine them into two. However, the form shown provides some computational convenience in that we can group the four dyads in two ways keeping either $\left(\underline{a}_{1}, \underline{a}_{2}\right)$ or $\left(\underline{b}_{1}, \underline{b}_{2}\right)$ explicit.

For this approximation we have $\left|e^{h \omega_{i}}-f_{2}\left(h \omega_{i}\right)\right|<0.07$ for all negative $h \omega_{i}$ (maximum 0.069 at $h \omega_{i}=-2.62$ ). This figure is small enough that results of high accuracy can be obtained by treating explicitly only the $\omega_{0}$ term in Eq. (8). Moreover if $\left|h \omega_{0}\right|$ is sufficiently below unity, none of the $\omega_{k}$ terms in Eq. (8) need be considered explicitly. 
The same complex factorization idea used here could be applied to the denominator of any higher order Padé approximation but there is not much advantage in going beyond $O\left(h^{3}\right)$, since there is an $0\left(h^{3}\right)$ error inherent in the assumption of constant reactivity and source during the time step as we will see in the next section. For this reason, only the two approximations above have been considered.

In summary, we developed in this section an efficient analytical method to invert polynomials of the matrix $A$ which has direct applicability to the Padé approximations. The method was applied to two specific cases, the Padé $(1,1)$ and the Pade $(2,0)$ approximations. Numerical characteristics of these approximations pertinent to their utilization in Eq. (8) were discussed. A concrete implementation for the method of Sec. $(2.3)$ is thus provided.

\subsection{Analysis of the Assumption of Constant Parameters}

The analysis done previously assumes constant reactivity and source during a time step. Taking them equal to their average value during the time interval hyields the smallest error. To see how good an approximation this is, and to support the statements made near the end of the previous section, an order of magnitude error analysis is now undertaken. Consider the following exact expansion for

$$
\begin{aligned}
& \Psi(t+h)=\underline{\Psi}_{1} \text { in terms of } \underline{\Psi}(t)=\underline{\Psi}_{0}: \\
& \qquad \underline{\Psi}_{1}^{\text {ex }}=\underline{\Psi}_{0}+h \underline{\Psi}_{0}+\frac{h^{2}}{2 !} \underline{\Psi}_{0}+\frac{h^{3}}{3 !} \underline{\Psi}_{0}+\ldots .
\end{aligned}
$$


By repeated use of $\underline{\Psi}_{0}=\mathrm{A}_{0} \underline{\Psi}_{0}+\underline{f}_{0}$, Eq. (10) becomes

$$
\begin{aligned}
\underline{\Psi}_{-1}^{e x}= & {\left[I+h A_{0}+\frac{h^{2}}{2 !}\left(A_{0}^{2}+\dot{A}_{0}\right)+\frac{h^{3}}{3 !}\left(A_{0}^{3}+2 \dot{A}_{0} A_{0}+A_{0} \dot{A}_{0}+\ddot{A}_{0}\right)+\ldots\right] \underline{\Psi}_{0}+} \\
& +\left[h \underline{f}_{0}+\frac{h^{2}}{2 !}\left(\dot{\Phi}_{0}+A_{0} \underline{f}_{0}\right)+\frac{h^{3}}{3 !}\left(\ddot{\dot{E}}_{0}+A_{0} \dot{\dot{\Phi}}_{0}+2 \dot{A}_{0} \underline{f}_{0}+A_{0}^{2} \underline{f}_{0}\right)+\ldots\right] .
\end{aligned}
$$

With the assumption of constant parameters we obtain the solution

$$
\underline{\Psi}_{-1}^{a p}=e^{h \bar{A}_{\Psi}} \underline{\Psi}_{0}+\bar{A}^{-1}\left(e^{h \bar{A}_{1}}-I\right) \overline{\underline{E}}_{,}
$$

as indicated in Eq. (2). Here, the bar indicates an average over the time step.

By using the series expansion for $e^{h \bar{A}}$ we then get

$$
\underline{\Psi}_{-1}^{\text {ap }}=\left[I+h \overline{\mathrm{A}}+\frac{h^{2}}{2 !} \overline{\mathrm{A}}^{2}+\frac{h^{3}}{3 !} \overline{\mathrm{A}}^{3}+\ldots\right] \underline{\Psi}_{0}+\mathrm{h}\left[I+\frac{h}{2 !} \overline{\mathrm{A}}+\frac{h^{2}}{3 !} \overline{\mathrm{A}}^{2}+\ldots\right] \underline{\underline{\mathrm{f}}} .
$$

But

$$
\overline{\mathrm{A}}=\frac{1}{\bar{h}} \int_{t_{0}}^{t_{0}+h} A(t) d t=A_{0}+\frac{h}{2 !} \dot{A}_{0}+\frac{h^{2}}{3 !} \ddot{A}_{0}+\ldots,
$$

and

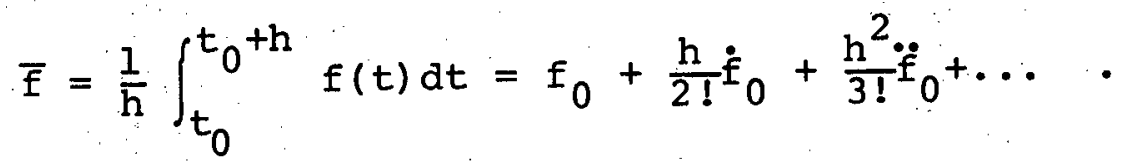

Substituting these expansions into Eq. (12) gives us

$$
\begin{aligned}
& \underline{\Psi}_{1}^{\mathrm{ap}}=\left[I+h \mathrm{~A}_{0}+\frac{h^{2}}{2 !}\left(\mathrm{A}_{0}^{2}+\dot{\mathrm{A}}_{0}\right)+\frac{h^{3}}{3 !}\left(\mathrm{A}_{0}^{3}+\frac{3}{2} \dot{\mathrm{A}}_{0} \mathrm{~A}_{0}+\frac{3}{2} \dot{A}_{0} \dot{\mathrm{A}}_{0}+\ddot{\mathrm{A}}_{0}\right)+\ldots\right] \underline{\Psi}_{0}+ \\
& +\left[h \underline{f}_{0}+\frac{h^{2}}{2 !}\left(\dot{\underline{f}}_{0}+A_{0} \underline{f}_{0}\right)+\frac{h^{3}}{3 !}\left(\ddot{\underline{\epsilon}}_{0}+\frac{3}{2} A_{0} \dot{\underline{x}}_{0}+\frac{3}{2} \dot{A}_{0} \underline{f}_{0}+A_{0}^{2} \underline{f}_{0}\right)+\ldots\right] \text {. }
\end{aligned}
$$

Then, from this result and Eq. (11) we obtain

$$
\underline{\psi}_{1}^{\mathrm{ex}}-\underline{\Psi}_{1}^{\mathrm{ap}}=\frac{\mathrm{h}^{3}}{12}\left[\dot{\mathrm{A}}_{0} \mathrm{~A}_{0}-\mathrm{A}_{0} \dot{\mathrm{A}}_{0}\right] \underline{\Psi}_{0}+\frac{\mathrm{h}^{3}}{12}\left[\dot{\mathrm{A}}_{0} \underline{\mathrm{f}}_{0}-\dot{\mathrm{A}}_{0} \underline{\dot{x}}_{0}\right]+0\left(\mathrm{~h}^{4}\right) \text {. }
$$


Note that this expression for the error still holds if $A(t)$ and $f_{(}(t)$ are known only through a three-term Taylor expansion, which requires only knowledge of $A, f$ and their first and second derivatives at the beginning of the time step. Thus, the assumption of constant parameters has a local error of $O\left(h^{3}\right)$ as stated in sec. (2.3) and, correspondingly, a global error of $0\left(\mathrm{~h}^{2}\right)$. This analysis is significant only for small h. It has been found that, in some practical cases where $\rho<\beta$, the error in $n(t)$ is essentially $0(h)$ for $h$ sufficiently larger than $1 / \omega_{I}$. 
Chapter 3

SOLUTION OF THE SPACE-DEPENDENT KINETICS EQUATIONS

\subsection{The Space-Dependent Kinetics Equations}

For problems in which changes in the shape of the neutron flux can no longer be neglected, the need arises to adopt a model that includes space and energy dependence. The multigroup diffusion model has been found adequate to represent the spatial effects for most practical problems. The more elaborate transport model has not been widely used for time dependent calculations, so far, due to its computational complexity.

In this chapter, we shall direct our attention to an implicit solution technique for the diffusion model, based on a method known as the "theta method", which we shall describe in Sec. (3.4). The properties of this method are considered in Sec. (3.6).

The model assumed to represent the space-time behavior of the neutron flux in a reactor is described by the time dependent multigroup diffusion equations and the associated delayed neutron precursor equations. In matrix form these equations are

$$
\begin{aligned}
\vec{\nabla} \cdot D \vec{\nabla} \Phi & -\Sigma \Phi+\sum_{i=1}^{I} \lambda_{i} \chi_{i} c_{i}=v_{0}^{-1} \dot{\phi} \\
\beta_{i} \underline{\Phi}^{T} \Phi-\lambda_{i} c_{i}=\dot{C}_{i} & , \quad(i=1,2, \ldots, I)
\end{aligned}
$$


where

$$
D=\operatorname{diag}\left[D_{g}(\vec{r}, t)\right], \quad v_{0}=\operatorname{diag}\left[v_{g}\right], \quad \Sigma=\left[\Sigma_{g g},(\vec{r}, t)\right],
$$

are $\mathrm{G} \times \mathrm{G}$ square matrices and

$$
\underline{f}=\operatorname{col}\left[v \Sigma_{f g}(\vec{r}, t)\right], \quad \underline{\phi}=\operatorname{col}\left[\phi_{g}(\vec{r}, t)\right], x_{i}=\operatorname{col}\left[x_{i g}\right],
$$

are G-vectors, with the following notation:

$$
\begin{aligned}
& g \text {. = index number for a neutron energy group } \\
& i \text { = index number for a delayed neutron group } \\
& \mathrm{G}=\text { total number of neutron energy groups } \\
& \text { I = total number of delayed neutron groups } \\
& \phi_{g}=\text { total scalar flux in group } g \\
& c_{i}=c_{i}(\vec{r}, t)=\text { precursor concentration for group } i \\
& v_{g}=\text { characteristic neutron velocity for group } g \\
& \mathrm{D}_{\mathrm{g}}=\text { diffusion constant for group } \mathrm{g} \\
& \Sigma_{g g^{\prime}}=\Sigma_{\mathrm{Tg}}(\vec{r} ; t) \delta_{g g^{\prime}}-\Sigma_{\text {sgg }}(\vec{r}, t)-(1-\beta) x_{0 g} \nu \Sigma_{f g^{\prime}}(\vec{r}, t) \\
& \Sigma_{T g}=\text { total macroscopic cross section for group } g \\
& \delta_{g^{\prime}}{ }^{\prime}=\text { Kroenecker delta } \\
& \Sigma_{\text {sgg }^{\prime}}=\text { macroscopic scattering cross section from group } \mathrm{g}^{\prime} \\
& \text { to group } \mathrm{g} \\
& \nu \Sigma_{f g}=\text { neutrons per fission } \times \text { macroscopic fission cross } \\
& \text { section for group } \mathrm{g} \\
& x_{0 g}=\text { fractional yield of neutrons in group } g \text {, per prompt } \\
& x_{i g}=\text { fractional yield of neutrons in group } 9 \text {, per decay } \\
& \text { of precursor } i \\
& B_{i}=\text { fractional yield of delayed neutrons in group } i \text {, }
\end{aligned}
$$


per fission

$\beta=\sum_{i=1}^{I} \beta_{i}=\underset{\text { per fission }}{\text { total fractional yield of delayed neutrons, }}$

$\lambda_{i}=$ decay constant for precursor $i$

We are assuming only one fissionable isotope and no external source present, for simplicity. However, extension of the method and its formalism for more than one fissionable isotope and an external source presents no particular difficulty.

Equation (1) can be written in the general matrix form

$$
\frac{d}{d t} \Psi=A(\vec{r}, t) \Psi(\vec{r}, t)
$$

where $\underline{\Psi}=\operatorname{col}\left[\Phi C_{1} C_{2} \ldots C_{I}\right]$, and

$$
A=\left[\begin{array}{ccccc}
v_{0}(\vec{\nabla} \cdot D \vec{\nabla}-\Sigma) & \lambda_{1} v_{0} \chi_{I} & \lambda_{2} v_{0} \chi_{2} & \cdots & \lambda_{I} v_{0} \chi_{I} \\
\beta_{1} \underline{f}^{T} & -\lambda_{1} & 0 & \cdots & 0 \\
\beta_{2} \underline{f}^{T} & 0 & -\lambda_{2} & \cdots & 0 \\
\vdots & \vdots & \vdots & \ddots & \vdots \\
\beta_{I} \underline{f}^{T} & 0 & 0 & \cdots & -\lambda_{I}
\end{array}\right]
$$

\subsection{Spatial Discretization}

Our main interest in this work is the study of a method to treat the time dependence. Therefore, we shall consider only the one-dimensional case, in which the position vector $\vec{r}$ is replaced by the coordinate $r$. The space dependence is 
treated by a simple finite-difference method: To do this, we divide the reactor in regions delimited by points $r_{k}$ in the $r$-axis and use the box integration procedure $(25)$ and thus obtain the finite-difference equations

$$
\begin{array}{r}
B_{k}^{\prime} \Phi_{k-1}+B_{k} \Phi_{k}+B_{k}^{\prime \prime} \Phi_{k+1}+\sum_{i=1}^{I} \lambda_{i} \chi_{i} C_{i k}=v_{0}^{-1} \dot{\Phi}_{k} \\
B_{i} \underline{f}_{k}^{T} \Phi_{k}-\lambda_{i} C_{i k}=\dot{C}_{i k}, \quad(i=1,2, \ldots, I)
\end{array}
$$

for $k=1,2, \ldots, k$, where $k$ is the total number of inner mesh points and

$$
\begin{gathered}
\underline{\phi}_{k}=\Phi\left(r_{k}, t\right) ; \quad c_{i k}=c_{i}\left(r_{k}, t\right) ; \\
B_{k+1}^{\prime}=B_{k}^{\prime \prime}=\frac{r_{k+1 / 2}^{q}}{\Delta r_{k} \Delta v_{k}} D\left(r_{k+1 / 2}, t\right) ; B_{k}=-\left[B_{k}^{\prime}+B_{k}^{\prime \prime}+\Sigma_{k}\right] ; \\
\Sigma_{k}=\frac{r_{k}^{q}}{2 \Delta v_{k}}\left[\Sigma\left(r_{k}^{+}, t\right) \Delta r_{k}+\Sigma\left(r_{k}^{-}, t\right) \Delta r_{k-1}\right] ; \\
\underline{f}_{k}=\frac{r_{k}^{q}}{2 \Delta v_{k}}\left[\underline{f}\left(r_{k}^{+}, t\right) \Delta r_{k}+\underline{f}\left(r_{k}^{-}, t\right) \Delta r_{k-1}\right] ; \\
\Delta r_{k}=r_{k+1}-r_{k} ; \Delta v_{k}=\frac{r_{k}^{q}}{2}\left(\Delta r_{k}+\Delta r_{k-1}\right) ; r_{k+1 / 2}=r_{k}+\frac{1}{2} \Delta r_{k}
\end{gathered}
$$

We have $q=0,1$, and 2 , for slab, cylindrical and spherical geometry, respectively. The + and - superscripts denote right and left limits of functions of $r$ at $r_{k}$, which may be different at material interfaces.

$$
\text { special relations apply to the boundary points } r_{0} \text { and }
$$


$\mathrm{r}_{\mathrm{K}+\mathrm{l}^{\prime}}$, which are derived from the boundary conditions at these points. These boundary conditions are usually homogeneous and of the Neumann or Dirichlet type.

We now group the elements of Eq. (3) in larger vectors and matrices by defining

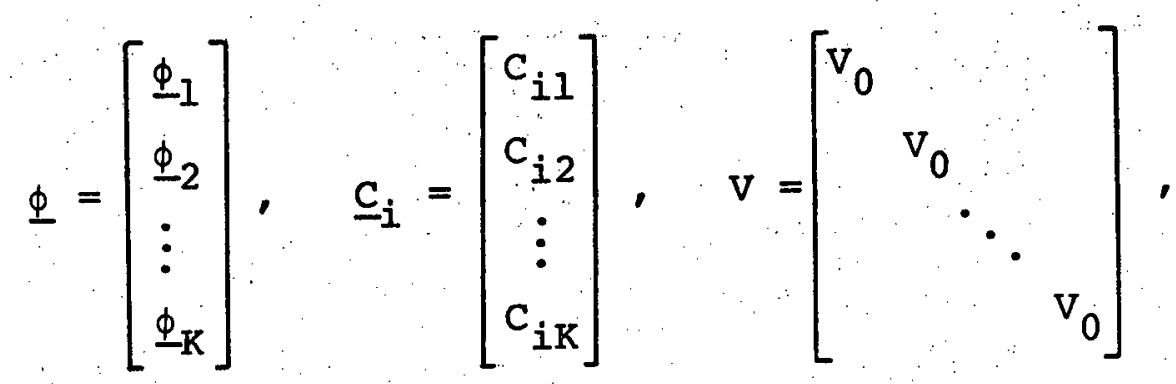

$$
\begin{aligned}
& \mathrm{L}=\left[\begin{array}{cccc}
\mathrm{B}_{1} & \mathrm{~B}_{1}^{\prime \prime} & & \\
\mathrm{B}_{2}^{\prime} & \mathrm{B}_{2} & \mathrm{~B}_{2}^{\prime \prime} & \\
& \ddots & \ddots & { }^{\mathrm{B}_{\mathrm{K}-1}^{\prime \prime}} \\
& & \mathrm{B}_{\mathrm{K}}^{\prime} & \mathrm{B}_{\mathrm{K}}
\end{array}\right]
\end{aligned}
$$

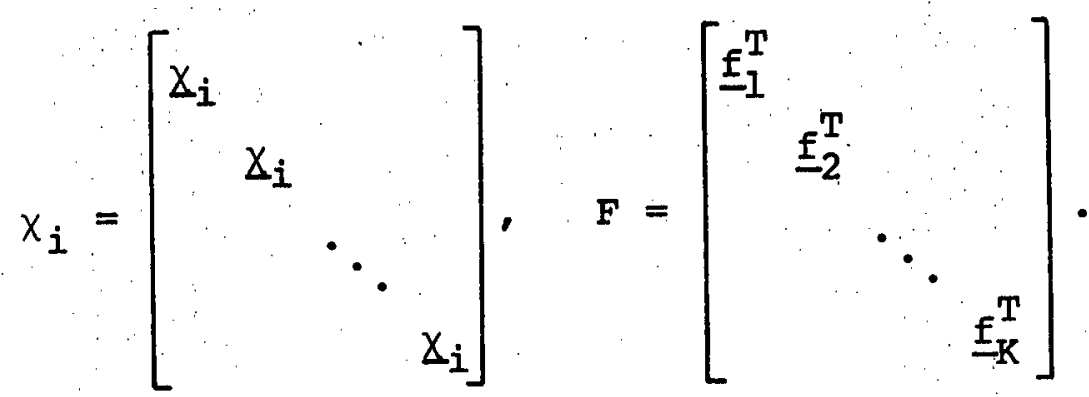

With these definitions, Eq. (3) becomes

$$
\begin{aligned}
& L_{\Phi}+\sum_{i=1}^{I} \lambda_{i} x_{i} \underline{C}_{i}=v^{-1} \dot{\phi} \\
& \beta_{i} F^{T}{ }^{T}-\lambda_{i} C_{i}=\dot{C}_{i} \quad(4-a)
\end{aligned}
$$

Note that $\Phi$ is a GK-vector and $\underline{C}_{i}$ is a K-vector. $\mathrm{L}$ and $V$ are $G K \times G K$ matrices, $\chi_{i}$ is a $G K \times K$ matrix, and $F$ is a $K \times G K$ 
matrix. The blocks of $L$ are GXG matrices, its off-diagonal blocks being diagonal. In the form shown above for $L$, we tacitly assumed the boundary conditions $\Phi_{0}=\Phi_{K+1}=0$, since all test cases will be for slab reactors and most of them will involve asymmetric transients.

In what follows, we shall prove several properties of the method adopted for the solution of Eqs. (4). Use of an even more compact notation will greatly facilitate such a task. Therefore, we write Eqs. (4) in the form

$$
\frac{d}{d t} \underline{\Psi}=H(t) \quad \underline{\Psi}(t)
$$

where

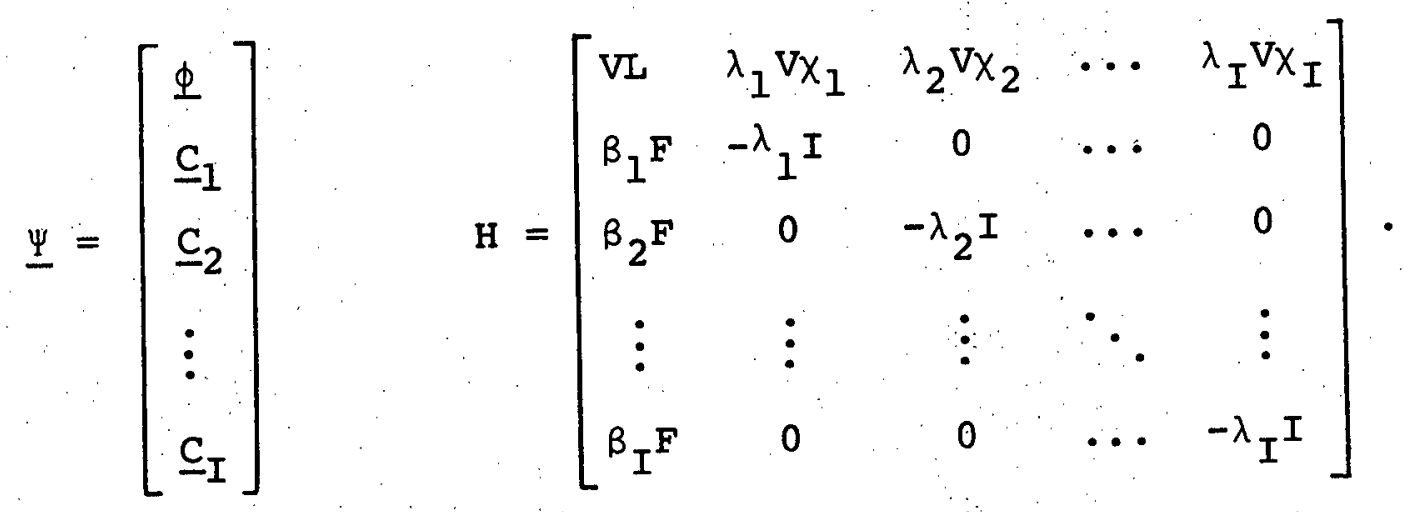

Note that the symbols $\underline{\Psi}$ and $\Phi$ are now supervectors that represent the vectors $\underline{\Psi}(\vec{r}, t)$ and $\Phi(\vec{r}, t)$ of Eq. (2) for all discrete points $r_{k}$. The use of the same symbols is intended to show the analogy between them, their precise meaning being clear from the context.

\subsection{Survey of Solution Methods}

The numerical solution of the kinetics equations is 
made very difficult by the fact that they form a "stiff". system, namely the characteristic times (or eigenvalues) involved span several orders of magnitude. (26) This eliminates from consideration explicit methods based on Taylor. series expansions in the time variable.

Several practical methods of solution for Eq. (2) exist, most based on its finite-difference formulation, given by Eq. (5) or by a higher-dimensional version of it. Here we mention a few of the most important.

To begin with, there are the nodal methods, (27) in which the reactor is divided into a few large regions, with some form of coupling between them specified. Another class of methods are the modal methods, in which the flux is expanded in a set of known modes or functions with coefficients determined by a variety of methods such as weighted residuals, least-squares or variational principles. $(28,29)$ Synthesis techniques are also widely used. They are actually special cases of the modal approach and emcompass a variety of schemes like time synthesis, (7) space-time synthesis, (30) and multichannel space-time systhesis. (31)

Quasi-static methods ${ }^{(6)}$ also form an important family of methods. These may be viewed as including the adiabatic and the point kinetics models, as special cases.

Finally we have the pure finite-difference methods. These lead to several different procedures for solving Eq. (5) : Among them are the matrix decomposition (GAKIN) meth- 
od, $(32,33)$ alternating-direction methods, $(34-36)$ for two and three dimensions, and the "theta" differencing (WIGLE, TWIGL) method. $(1,2,37,38)$ As mentioned before, it is to this last method that we shall direct our attention in the present work.

A good survey of the above methods, as well as others, is provided by Ref. (39). It includes both descriptions and references:

\subsection{Derivation of the Theta-Differenced Equations}

The theta method is an implicit method involving the use of special parameters (thetas) intended to increase the accuracy of the difference equations for the time step at hand: It can be viewed as a generalization of the Crank-Nicholson method and includes, as special cases, both the totally explicit and the totally implicit methods. Being in general. implicit, it involves inversion of large matrices, which, in turn, requires relatively large amounts of computation time, per time step. On the other hand, if appropriate values of the thetas can be found, the method is able to take comparatively large time steps with a reasonably good accuracy. One purpose of the present thesis is to improve the current methods $(1,2,37,38)$ by which thetas are found. We shall attempt to find some efficient systematic procedure for generating these parameters, which will be applicable under all transient conditions.

The equations for the "theta method" can be derived by 
integrating both sides of Egs. (4) from $t$ to $t+h$, thus obtaining

$$
\begin{aligned}
& \mathrm{v}^{-1}\left(\Phi_{1}-\Phi_{0}\right)=\int_{0}^{h} L(t+s) \Phi(t+s) d s+\sum_{i=1}^{I} \lambda_{i} X_{i} \int_{0}^{h} \underline{C}_{i}(t+s) d s \\
& \underline{c}_{i 1}-\underline{C}_{i 0}=\beta_{i} \int_{0}^{h} F(t+s) \Phi(t+s) d s-\lambda_{i} \int_{0}^{h} \underline{c}_{i} d s, \quad(i=1,2, \ldots, I)
\end{aligned}
$$

where the subscripts 0 and 1 refer to functions at $t$ and $t+h$, respectively. Then the approximations

$$
\begin{aligned}
& \frac{1}{h} \int_{0}^{h} L(t+s) \Phi(t+s) d s=\theta_{0} L_{1} \Phi_{1}+\left(1-\theta_{0}\right) L_{0} \Phi_{0} \\
& \frac{1}{h} \int_{0}^{h} F(t+s) \Phi(t+s) d s=\theta_{0} F_{1} \Phi_{1}+\left(1-\theta_{0}\right) F_{0} \Phi_{0} \\
& \frac{1}{h} \int_{0}^{h} \underline{c}_{i}(t+s) d s=\theta_{i} \underline{C}_{i 1}+\left(1-\theta_{i}\right) C_{i 0} \quad(i=1,2, \ldots, I)
\end{aligned}
$$

are made, where the thetas will be determined so as to yield a good solution under the condition that $0<\theta_{i} \leq I$ ( $i=0$ to $I$ ). Substituting the approximations (7) in Eq. (6) yields

$$
\begin{aligned}
\frac{1}{h} \mathrm{~V}^{-1}\left(\Phi_{1}-\Phi_{0}\right)=\left[\theta_{0} L_{1} \Phi_{1}+\left(1-\theta_{0}\right) L_{0} \Phi_{0}\right]+ & \\
& +\sum_{i=1}^{I} \lambda_{i} x_{i}\left[\theta_{i} \underline{C}_{i 1}+\left(1-\theta_{i}\right) \underline{C}_{i 0}\right] \\
\frac{1}{h}\left(\underline{C}_{i 1}-\underline{C}_{i 0}\right)=\beta_{i}\left[\theta_{0} F_{1} \Phi_{1}\right. & \left.+\left(1-\theta_{0}\right) F_{0} \Phi_{0}\right]- \\
& -\lambda_{i}\left[\theta_{i} \underline{C}_{i 1}+\left(1-\theta_{i}\right) \underline{C}_{i 0}\right]
\end{aligned}
$$

In principle, Eqs. (8) would yield the exact solution, if we used the proper thetas, one for each space point and neutron energy group. However, knowledge of such thetas 
would imply knowledge of the exact solution, which is already what we are looking for Thus approximations have to be made in order to implement Egs. (8). Specifically, we assume a single theta parameter for all spatial points and, for the flux, the same parameter also for all neutron energy groups.

In the WIGLE approach $(1,37)$ a different theta is used for each neutron energy group, but only one theta is assumed for all precursors, namely $\theta_{i}=\theta_{d}(i=1,2, \ldots, I)$. The thetas are assumed to be space-independent and are estimated from a two-group point calculation (WIGLE-type codes are limited to two energy groups). Drastic approximations are made in the point equations, in order to compute these thetas. In TWIGL, $(2,38)$ the thetas for the precursors are all chosen as $\theta_{i}=1 / 2 \quad(i=1,2, \ldots, I)$ and $\theta_{0}$ is left as in input parameter, the same for all energy groups and space points.

In the present work, the idea is to use an independent ${ }^{\theta_{i}}$ for each precursor and the flux, since they have distinctly different characteristic times. As mentioned before, the same theta is used for all space points (as in WIGLE and TWIGL) and, in the case of the flux, also for all neutron energy groups. It is felt that, since the energy coupling is stronger than the spatial coupling, at least for thermal reactors, there is no justification in taking a different integration parameter for each energy group while using only one for all the points of space.

We shall examine, in this thesis, the feasibility of 
using an accurate point kinetics calculation as a means of finding the thetas so that Eqs. (8) will be accurate in an integral sense. The details of this procedure are spelled out in the next section.

To get the time advancement algorithm we first define the total (prompt + delayed) net production operator

$$
M(t)=L(t)+\sum_{i=1}^{I} \beta_{i} X_{i} F(t)
$$

Solving Eq. (8-b) for $\underline{C}_{i l}$ and substituting in Eq. (8-a) yields to new equations,

$$
\begin{aligned}
& {\left[M_{1}-\frac{i}{h \theta_{0}} V^{-1}-\sum_{i=1}^{I} \beta_{i} x_{i} \frac{e_{i}^{\prime}}{\theta_{0}} F_{1}\right] \Phi_{1}+\left[\frac{1-\theta_{0}}{\theta_{0} M_{0}}+\frac{i}{h \theta_{0}} V^{-1}-\right.} \\
& \left.-\sum_{i=1}^{I} \beta_{i} X_{i} \frac{\tau_{i}-\theta !}{\theta_{0}} F_{0}\right] \Phi_{0}+\sum_{i=1}^{I} \lambda_{i} \frac{\tau_{i}}{\theta_{0}} x_{i} C_{i 0}=0 \\
& \underline{C}_{i 1}=\left(1-h \lambda_{i} \tau_{i}\right) \underline{C}_{i 0}+h \beta_{i} \theta_{i}^{\prime} F_{1} \Phi_{1}+h \beta_{i}\left(\tau_{i}-\theta_{i}^{\prime}\right) F_{0} \Phi_{0},
\end{aligned}
$$

where

$$
\begin{aligned}
\tau_{i} & =\frac{1}{1+h \lambda_{i} \theta_{i}} \\
\theta_{i} & =\frac{\theta_{0}}{1+h \lambda_{i} \theta_{i}}
\end{aligned}
$$

This procedure will henceforth be called Method 1 . We can also take a slightly different approach which we shall call Method 2. Instead of first approximating the equations and then solving for $\underline{C}_{i l}$ we can first solve $\mathrm{Eq}$. (4-b) for $\underline{c}_{i}$, substitute in Eq. (4-a), and then integrate, thus obtaining 


$$
\begin{gathered}
V^{-1}\left(\Phi_{1}-\phi_{0}\right)=\int_{0}^{h} M(t+s) \Phi(t+s) d s-\sum_{i=1}^{I} \beta_{i} X_{i} \int_{0}^{h} e^{-\lambda}(h-s) \\
\cdot F(t+s) \Phi(t+s) d s+\sum_{i=1}^{I} X_{i}\left(1-e^{-h \lambda_{i}} C_{i 0}\right.
\end{gathered}
$$$$
\underline{c}_{i 1}=e^{-h \lambda} \underline{C}_{i 0}+\beta_{i} \int_{0}^{h} e^{-\lambda{ }_{i}(h-s)} F(t+s) \Phi(t+s) d s,
$$

which we call the precursor integrated equations.

We now make the approximations

$$
\begin{aligned}
& L(t+s) \Phi(t+s)=\theta(s) L_{1} \Phi_{1}+[1-\theta(s)] L_{0} \Phi_{0} \\
& F(t+s) \Phi(t+s)=\theta(s) F_{1} \Phi_{1}+[1-\theta(s)] F_{0} \Phi_{0}
\end{aligned}
$$

for $0 \leq s \leq h$, where $\theta(s)$ is a space-independent function to be determined so as to yield good results, subject to the conditions $\theta(0)=0, \theta(h)=1$, and $0 \leq \theta(s) \leq 1$ in the above interval for s. Taking $\theta(s)=s / h$ means that all reaction rates are assumed to vary linearly between their initial and final values. This approach has been used in Ref. (47). By introducing Eq. (13) in Eq. (12) and carrying out the integrations we get again Eqs. (10) where now

$$
\begin{aligned}
& \tau_{i}=\frac{1}{h} \int_{0}^{h} e^{-\lambda_{i}(h-s)} d s=\frac{1-e^{-h \lambda_{i}}}{h \lambda_{i}} \\
& \theta_{i}=\frac{1}{h} \int_{0}^{h} \theta(s) e^{-\lambda_{i}(h-s)} \text { ds } \\
& \theta_{0}=\frac{1}{h} \int_{0}^{h} \theta(s) d s .
\end{aligned}
$$


We impose an additional condition for $\theta(s)$, namely that $0<\theta_{0} \leq 1$

As in Method 1, we shall, in the next section, try to apply point kinetics to find $\theta(s)$ or directly the parameters of Eq. (14)

Note that we have from either Eq. (11) or (14) the following results

$$
\begin{aligned}
& \theta_{i}<\theta_{0} \\
& \theta_{i} \leq \tau_{i}
\end{aligned}
$$

as can easily be inferred from their definitions and the restrictions placed on the thetas. These results will be useful' later.

$$
\text { From Eqs. (11) and (14) it can be shown that the two }
$$
methods are identical if

$$
\frac{1}{h} \int_{0}^{h} \theta(s) e^{\lambda_{i} s} d s=\left[\frac{1}{h} \int_{0}^{h} \theta(s) d s\right]\left[\frac{1}{h} \int_{0}^{h} e^{\lambda_{i} s} d s\right],
$$

provided $\theta_{0}$ is the same for both methods and provided we take

$$
\theta_{i}=\frac{1}{\left(-h \lambda_{i}\right)}-\frac{1}{e^{\left(-h \lambda_{i}\right)}-1}
$$

Equation (16) holds exactly only for constant $\theta(\mathrm{s})$, but it becomes an increasingly better approximation as $h \lambda_{i}$ becomes small. 
3.5 Determination of the Parameters Theta

As indicated in the previous section, a possible way to get a set $\left\{\theta_{i}\right\}$ or a function $\theta(s)$ is through a corresponding point kinetics calculation, which makes Eqs. (8) or (12) "exact". in an integral sense.

The point kinetics problem is obtained by reducing all space-energy dependent quantities to their point equivalent counterparts. This is done simply by multiplying the quantities of interest by a weight function of space and energy and integrating over the domain of interest of these variables. In the multigroup, space-discretized formulation such a procedure corresponds to a scalar product of a weight vector $\underline{w}$ and the vector quantity under consideration. We thus define

$$
\begin{array}{ll}
n(t)=\underline{w}^{T} v^{-1} \Phi, & c_{i}=\frac{w^{T}}{\beta} x_{i} \underline{c}_{i}, \\
\alpha(t)=\frac{1}{n} \underline{w}^{T} L \Phi, & \mu_{i}(t)=\frac{{ }_{i}}{n} \underline{w}^{T} x_{i} F \Phi,
\end{array}
$$

where $\underline{w}$ is a GK-vector given by

$$
\underline{w}=\operatorname{col}\left[\begin{array}{llll}
\underline{w}_{1} & \underline{w}_{2} & \cdots & \underline{w}_{K}
\end{array}\right]
$$

with the G-vectors

$$
\underline{w}_{k}=\Delta v_{k} \times \operatorname{col}\left[w_{1 k} w_{2 k} \cdots w_{G k}\right]
$$

With this choice of $\underline{w}$, the scalar products in Eq. (17) correspond to a trapezoidal integration in space. One choice for the elements $w_{g k}$ can be the positive solution 
$\phi_{g}^{*}\left(r_{k}\right)$ of the equation

$$
\mathrm{M}^{\mathrm{T}}(0) \phi^{*}=0
$$

which is the discretized equation for the adjoint in the initial steady-state. More simply we could also take $\underline{w}=$ constant. other possibilities would be to use $\underline{w}=\Phi(t)$ itself or to use at every time the adjoint of the perturbed state, made critical by dividing the matrix $F$ by the appropriate scalar. This last procedure would probably yield the best results but would be too time-consuming.

An approximation has to be introduced at this point in order to calculate $\alpha(t)$ and $\mu(t)$. We assume that the shape of the flux $\Phi$ remains constant during the time step $h$, and is given by its value at the beginning of that time step. Note that this approximation of constant shape is used only to calculate the thetas and does not imply a similar assumption for the space-dependent calculations. We thus have that

$$
\alpha(t)=\frac{1}{n(t)} \underline{w}^{T} L(t) \Phi_{0}, \quad \mu_{i}(t)=\frac{\beta_{i}}{n(t)} \underline{w}^{T} \chi_{i} F(t) \Phi_{0} .
$$

With these definitions, Eq. (5) will reduce to the point kinetics Eq. (2.1). This equation can be solved by the method of Chap. 2 yielding $n_{1}$ and $c_{i l}$ as functions of $\mathrm{n}_{0}$ and $\mathrm{c}_{\mathrm{i} 0}$.

Introducing Eqs. (17) into the theta-differenced Eqs. (8) yields 


$$
\begin{aligned}
& \frac{n_{1}-n_{0}}{h}=\left[\theta_{0} \alpha_{1} n_{1}+\left(1-\theta_{0}\right) \alpha_{0} n_{0}\right]+\sum_{i=1}^{I} \lambda_{i}\left[\theta_{i} c_{i 1}+\left(1-\theta_{i}\right) c_{i 0}\right] \\
& \frac{c_{i 1}-c_{i 0}}{h}=\left[\theta_{0} \mu_{i 1} n_{1}+\left(1-\theta_{0}\right) \mu_{i 0} n_{0}\right]-\lambda_{i}\left[\theta_{i} c_{i 1}+\left(1-\theta_{i}\right) c_{i 0}\right]
\end{aligned}
$$

Calculating the $\mathrm{n}_{1}$ and $\mathrm{c}_{i l}$ from $\mathrm{Eq} \cdot(2.1)$ and using these values in Eq. (18) allows us to find the desired theta parameters: A detailed account of this procedure is given in Appendix B. For the second approach, using the precursor integrated equations, we assume that $L \Phi$ and $F \Phi$ go from their initial to final values at the same rate with which the amplitude function $n(t)$ foes from $n_{0}$ to $n_{1}$. Thus, $\theta(s)$ is such that

$$
n(t+s)=\theta(s) n_{1}+[1-\theta(s) \cdot] n_{0}
$$

from which we get

$$
\theta(s)=\frac{n(t+s)-n_{0}}{n_{1}-n_{0}}
$$

The function $n(t)$ is obtained by solving Eq. (2.1). With this $\theta(s)$ we can then find the parameters $\theta_{0}$ and $\theta_{i}$ of Eq. (14) (see Appendix B for a detailed description of the procedure used.)

of course the thetas may also be chosen without the use of a point kinetics formulation. For instance we may simply pick any of the thetas to be $\theta_{i}=1$ or $\theta_{i}=\frac{1}{2}$ or 


$$
\theta_{i}=\frac{1}{h \omega_{0}}-\frac{1}{e^{h \omega_{0}}} \text {, }
$$

if the reactor is on an asymptotic period $\omega_{0}$. Also $\theta$ (s) could be picked as $\theta(s)=s / h$ or $\theta(s)=1$ (step function) or, in the asymptotic period case,

$$
\theta(s)=\frac{e^{s \omega_{0}}-1}{e^{h \omega_{0}}-1}
$$

\subsection{Properties of the Solution Method}

In this section, we shall analyze several properties of the two methods of solution, as presented in $\mathrm{Sec}$ (3.4). The methods will first be shown to be consistent and stable, for the case in which the reactor parameters are constant in time.

Consistency and stability are necessary and sufficient conditions for the convergence of the finite-difference solution, by Lax's theorem. (40) These two properties have been shown to hold for Method 1 , in the case of constant spatial mesh ${ }^{(41)}$ and can easily be extended to Method 2 for such a case. Here we show that they also hold when the spatial mesh size is shrunk to zero, together with the time step size, provided that

$$
\frac{h}{(\Delta r)^{2}}=\text { const. , } \quad \theta_{0} \geq \frac{1}{2} \text {. }
$$

The two methods will also be shown to conserve the steady-state for any thetas, and to be asymptotically exact, 
if asymptotic thetas are used. Asymptotic stability is also examined. Finally, the solvability of the time advancement equations (10) will be analyzed.

In the case of constant spatial mesh it has been shown (41)

that the global truncation error for Method $I$ is $0(h)$ for a general theta matrix and is $0\left(h^{2}\right)$ if $\theta=\frac{1}{2} I$. Similar$1 y$, for Method 2 it can be readily shown that the global truncation error is $O(h)$ for a general $\theta(s)$ and $O\left(h^{2}\right)$ if $\theta(\mathrm{s})$ is such that $\theta_{0}=\frac{1}{2}$.

The proofs of consistency and stability will be carried in the $I_{2}$ norm given by

$$
\|\underline{u}(r)\|=\left[\int_{V}|\underline{u}(r)|^{2} d v\right]^{1 / 2} .
$$

For functions defined only at certain discrete points $r_{k}$, some interpolation scheme is assumed for the intermediate points. For an operator $A$ the $L_{2}$ norm is defined as

$$
\|\mathrm{A}\|=\max _{\|\underline{\mathrm{u}}\|=1}\|\underline{\mathrm{u}}\|
$$

In the Appendix $C$ we show that, for a discrete operator or matrix $\mathrm{H}$,

$$
\mathrm{k}\|\mathrm{H}\| \mathbf{}\left\|^{\prime}\right\| \mathrm{H}\|\leq \mathrm{K}\| \mathrm{H} \| \text { ' }
$$

where \|\|$^{\prime}$ is the Euclidean norm, (23) and $k, k$ are fixed, positive constants. The above relation means that the $\mathrm{L}_{2}$ norm and the Euclidean norm of a matrix are equivalent so that all properties of boundedness of matrices in one norm hold for the other norm. 
When stating that certain matrices have bounded norms we shall be tacitly using the following

THEOREM: (34) A family of matrices $\mathrm{H}_{\mathrm{N}}$ of varying dimension $\mathrm{N}$ having at most $\mathrm{n}<\mathrm{N}$ nonzero elements in each row or column, $\mathrm{n}$ being constant for all $\mathrm{N}$, has a uniformly bounded Euclidean norm if the individual elements of the matrices $\mathrm{H}_{\mathrm{N}}$ are uniformly bounded for all $\mathrm{N}$.

It will be convenient to rederive the iteration algorithms in a more compact form to carry out the proofs that follow. To this end we consider $\mathrm{H}$ as composed of two terms

$$
H(t)=Q+R(t)
$$

We then write Eq. (5) in the form

$$
\frac{d}{d t} \underline{\Psi}-Q \underline{\Psi}(t)=R(t) \underline{\Psi}(t)
$$

a solution of which is given by

$$
\underline{\Psi}_{1}=e^{h Q_{\Psi}}+\int_{0}^{h} e^{(h-s) Q} R(t+s) \underline{\Psi}(t+s) d s,
$$

where the subscripts 0 and 1 denote quantities evaluated at $t$ and $t+h$, respectively.

For Method 1 we have $Q=0$ and $R=H$, and we make the approximation

$$
\frac{1}{h} \int_{0}^{h} R(t+s) \underline{\Psi}(t+s) d s=\theta R_{1} \underline{\Psi}_{1}+(I-\theta) R_{0} \underline{\Psi}_{0}
$$

of Eqs. (7), where 


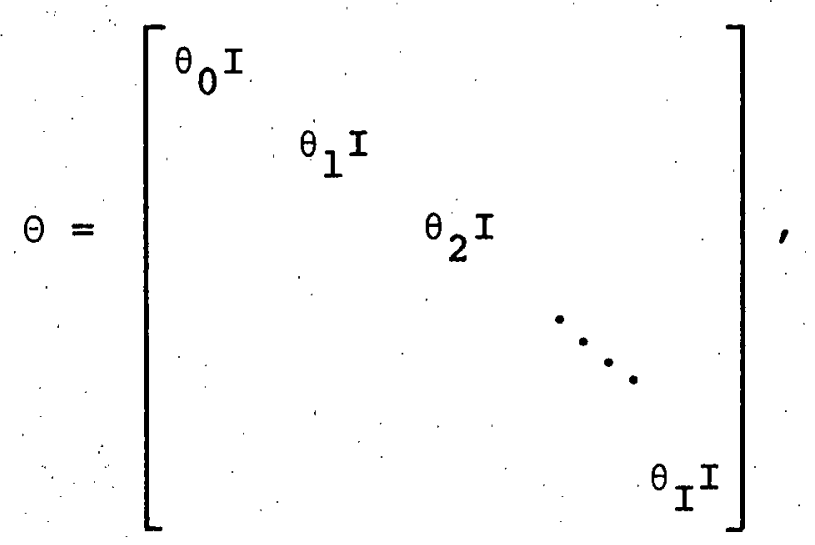

with the first $I$ being $G K \times G K$ and the others being $K \times K$.

For Method 2, in order to get Eq. (12), we must have

$$
Q=\left[\begin{array}{ccccc}
0 & \lambda_{1} \mathrm{Vx}_{1} & \lambda_{2} \mathrm{Vx}_{2} & \cdots & \lambda_{I} \mathrm{Vx}_{I} \\
0 & -\lambda_{1} \mathrm{I} & 0 & \cdots & 0 \\
0 & 0 & -\lambda_{2} \mathrm{C} & \cdots & 0 \\
\vdots & \vdots & \vdots & \ddots & \vdots \\
0 & 0 & 0 & \cdots & -\lambda_{I}
\end{array}\right],
$$

$$
R=\left[\begin{array}{ccccc}
\mathrm{VL} & 0 & 0 & \cdots & 0 \\
\beta_{I} F & 0 & 0 & \cdots & 0 \\
\beta_{2} F & 0 & 0 & \cdots & 0 \\
\vdots & \vdots & \vdots & \ddots & \vdots \\
\beta_{I} F & 0 & 0 & \cdots & 0
\end{array}\right],
$$

and the approximation is made that (see Eq. (13))

$$
R(t+s) \underline{\Psi}(t+s)=\theta(s) R_{1} \Psi_{1}+[1-\theta(s)] R_{0} \Psi_{0} .
$$


Introducing either of these approximations, Eq. (24) or (27), in Eq. (23) yields

$$
\begin{aligned}
\underline{\Psi}_{1} & =\left[I-h \Theta \mathrm{R}_{1}\right]^{-1}\left[\mathrm{e}^{\mathrm{h}}+\mathrm{h}(\Lambda-\Theta) \mathrm{R}_{0}\right] \underline{\Psi}_{0} \\
& =E(h) \underline{\Psi}_{0},
\end{aligned}
$$

where $E(h)$ is the time advancement matrix. For Method 1, $\theta$ is given by Eq. (25) and for Method 2, we have

$$
\theta=\frac{1}{h} \int_{0}^{h} e^{(h-s) Q} \theta(s) d s
$$

For both methods, $\Lambda$ is given by

$$
\Lambda=\frac{1}{h} \int_{0}^{h} e^{(h-s) Q} d s,
$$

which reduces to $\Lambda=I$ for Method 1 . Equation (28) is equivalent to the system of Eqs. (10), as can be verified for both methods. From Eq. (30) we can readily derive the identity

$$
e^{h Q}-I-h \Lambda Q=0
$$

valid for any $Q$.

Equation (28) will be the one used for the demonstration of the several properties of both solution methods presented here. We shall assume constant properties during a time step in most of the remaining of this section either for simplicity, to show consistency and stability, or by necessity, when analyzing steady-state and asymptotic behavior. 


\subsubsection{Consistency}

The property of consistency simply implies that the truncation error of the finite-difference formulation tends to zero, as the time step size tends to zero: Mathematical1y we must have ${ }^{(40)}$

$$
\lim _{h \rightarrow 0}\left\|\frac{\underline{\Psi}_{1}-E(h) \underline{\Psi}_{0}}{h}\right\|=0
$$

for any $\underline{\Psi}(\vec{r}, t)$ which is a genuine solution of $\mathrm{Eq}$. (2). The convention is made that a matrix operating on a continuous function of $r$ is actually operating on the corresponsing vector obtained from the function values at the discrete points $r_{k}$. From the properties of norms we have, using Eq. (28),

$$
\left\|\frac{\underline{\Psi}_{1}-E(h) \underline{\Psi}_{0}}{h}\right\| \leq\left\|[I-h \Theta R]^{-1}\right\| \cdot\left\|\frac{[I-h \Theta R] \underline{\Psi}_{1}-\left[e^{h Q+h(\Lambda-\theta) R] \underline{\Psi}_{0}}\right.}{h}\right\| .
$$

We shall prove afterwards that

$$
\lim _{h \rightarrow 0}\left\|[I-h \Theta R]^{-1}\right\|
$$

exists, if $h /(\Delta r)^{2}=$ const as $h \rightarrow 0$.

Now, it remains to be shown that

$$
\lim _{h \rightarrow 0}\left\|\frac{[I-h \theta R] \underline{\Psi}_{1}-\left[e^{h Q}+h(\Lambda-\theta) R\right] \underline{\Psi}_{0}}{h}\right\|=0 .
$$

We have $\underline{\Psi}_{1}=\underline{\Psi}_{0}+h \underline{\dot{\Psi}}(t+\varepsilon h)$, for some $\varepsilon$ in the interval $[0,1]$. Recalling that $\mathrm{H}=Q+\mathrm{R}$ and using Eq. (31), the expression whose norm is being taken in Eq. (32) becomes 


$$
[I-h \Theta H+h \Theta Q] \underline{\dot{\Psi}}(t+\varepsilon h)-\Lambda H \underline{\Psi}_{0} \text {. }
$$

Now we use Eq. (2) and the result $H=A+0\left(\Delta r^{n}\right)$, where $\mathrm{n}$ is the order of accuracy of the spatial discretization, usually $n=2$. Then the expression above becomes

$$
\begin{aligned}
& {\left[I-h \Theta A-0\left(\Delta \mathrm{r}^{\mathrm{n}}\right) h \Theta+h \Theta Q\right] \underline{\Psi}(t+\varepsilon h)-\Lambda \underline{\Psi}_{0}-0\left(\Delta \mathrm{r}^{\mathrm{n}}\right) \underline{\Lambda}_{-}=} \\
= & {[I-0(h) A+0(h)] \underline{\Psi}(t+\varepsilon h)-[I+0(h)] \underline{\Psi}_{0}+0\left(h^{\mathrm{H} / 2}\right) \underline{\Psi}_{0}, }
\end{aligned}
$$

where we have used the facts that $\theta$ and $Q$ have bounded norms, $\Lambda=I+O(h)$ and $\Delta r^{2} / h=$ const. From Eq. (2), $A \dot{\Psi}=\ddot{\Psi}$, for $A$ constant in time. Also we have

$$
\underline{\dot{\Psi}}(t+\varepsilon h)=\dot{\Psi}_{0}+\varepsilon h \underline{\ddot{\Psi}}\left(t+\varepsilon^{\prime} h\right),
$$

for some $\varepsilon^{\prime}$ in the interval $[0, \varepsilon]$. Using these two equations and the fact that $\underline{\Psi}, \underline{\dot{\Psi}}$, and $\underline{\dot{\Psi}}$ have bounded $I_{2}$ norms in the interval $(t, t+h)$, we can reduce the expression under consideration to $0(\mathrm{~h})+0\left(\mathrm{~h}^{\mathrm{n} / 2}\right)$. Thus Eq. (32) is proved.

We now show that $\left\|[I-h \Theta R]^{-1}\right\|$ is bounded as $h \rightarrow 0$. Let us write $R=R_{D}+R^{\prime}$ where $R_{D}$ contains only the diffusion terms (of the $V L$ matrix) and $R^{\prime}$ contains all the other elements of $R$. Then

$$
\begin{aligned}
{[I-h \Theta R]^{-1} } & =\left[I-\left(I-h \Theta R_{D}\right)^{-1} h \Theta R^{\prime}\right]^{-1}\left[I-h \Theta R_{D}\right]^{-1} \\
& =\left[I-\left(I-h \theta_{0} R_{D}\right)^{-1} h \Theta R^{\prime}\right]^{-1}\left[I-h \theta_{0} R_{D}\right]^{-1},
\end{aligned}
$$

since $\theta R_{D}=\theta_{0} R_{D}$ 
The matrix $R_{D}$ is real, symmetric, diagonally dominant, and has non-positive diagonal elements, therefore, the eigenvalues of $R_{D}$ are all real and non-positive. (42) Thus, since the Euclidean norm of a symmetric matrix is equal to its spectral radius, (23)

$$
\left\|\left[I-h \theta_{0} R_{D}\right]^{-1}\right\|^{\prime}=\rho\left(\left[I-h \theta_{0} R_{D}\right]^{-1}\right)=\max _{n}\left|\frac{1}{1-h \theta_{0} \gamma_{n}}\right|=1,
$$

since zero is also an eigenvalue of $R_{D}$. Here, $p$ denotes the spectral radius, and $\gamma_{n}$ are the eigenvalues of $R_{D}$.

With this result we have

$$
\left\|[I-h \ominus R]^{-1}\right\|^{\prime} \leq\left\|\left[I-\left(I-h \theta_{0} R_{D}\right)^{-1} h h^{\prime}\right]^{-1}\right\|^{\prime} \leq \frac{1}{I-\beta}
$$

where

$$
\beta=\left\|\left[I-h \theta R_{D^{\prime}}\right]^{-1} h \theta R^{\prime}\right\|^{\prime} \leq h\left\|\theta R^{\prime}\right\|^{\prime}<1
$$

for $h$ sufficiently small, since $\theta$ and $R^{\prime}$ are bounded as $h \rightarrow 0$.

Using the equivalence of the $\mathrm{I}_{2}$ and the Euclidean norms we then conclude that $\left\|[I-h \ominus R]^{-1}\right\|$ is indeed bounded as $h \rightarrow 0$.

\subsubsection{Stability}

The property of stability assures us that the error in the solution will not grow without bounds as $h \rightarrow 0$. Recalling the equivalence of the $\mathrm{I}_{2}$ and the Euclidean norms, a sufficient condition for stability is that $(40)$

$$
\|E(h)\|^{\prime}=1+0(h)
$$


We again factor $R$ in two terms $R_{D}+R^{\prime}$ as seen in Sec. (3.6.1), where $R_{D}$ contains only the diffusion terms. Then we write

$$
\begin{aligned}
E(h)= & {\left[I-\left(I-h \Theta R_{D}\right)^{-1} h \Theta R^{\prime}\right]^{-I}\left[I-h \Theta R_{D}\right]^{-1} } \\
& \cdot\left[I+h(I-\Theta) R_{D}+\left(e^{h Q}-I\right)+h(\Lambda-\Theta) R^{\prime}-h(I-\Lambda) R_{D}\right]
\end{aligned}
$$

Now we use the following equations

$$
\begin{aligned}
e^{h Q}-I & =0(h) \\
h[\Lambda-\theta] \cdot R^{\prime} & =0(h) \\
h[I-\Lambda] \cdot R_{D} & =0\left(h^{2}\right) R_{D}=0(h)
\end{aligned}
$$

which can be seen to hold since $Q, \Lambda, \theta, R^{\prime}$, and $h R_{D}$ have bounded norms and $\|\Lambda-I\|=O(h)$. Thus, since $\left[I-h \Theta R_{D}\right]^{-1}$ is also bounded, as shown in Sec. (3.6.1), we have

$$
\begin{aligned}
E(h) & =[I-0(h)]^{-1}\left[I-h \theta_{0} R_{D}\right]^{-1}\left[I+h\left(I-\theta_{0}\right) R_{D}+0(h)\right] \\
& =\left[I-h \dot{\theta}_{0} R_{D}\right]^{-1}\left[I+h\left(I-\theta_{0}\right) R_{D}\right]+0(h)
\end{aligned}
$$

so that

$$
\|E(h)\|^{\prime}=\left\|\left[I-h \theta_{0} R_{D}\right]^{-1}\left[I+h\left(I-\theta_{0}\right) R_{D}\right]\right\|^{\prime}+0(h)
$$

But since $R_{D}$ is symmetric and has only real non-positive eigenvalues, including zero eigenvalues, as we have seen in Sec. (3.6.1), we may write

$$
\begin{aligned}
\|E(h)\| 1 & =\rho\left(\left[I-h \theta_{0} R_{D}\right]^{-1}\left[I+h\left(1-\theta_{0}\right) R_{D}\right]\right)+0(h)= \\
& =\max _{n}\left|\frac{1+h\left(1-\theta_{0}\right) \gamma_{n}}{1-h \theta_{0} \gamma_{n}}\right|+0(h)=1+0(h),
\end{aligned}
$$


provided $\theta_{0} \geq \frac{1}{2}$. Again, $\rho$ denotes the spectral radius, and $\gamma_{n}$ are the eigenvalues of $R_{D}$.

\subsubsection{Steady-State Behavior}

We will now show that, given steady-state properties and a steady-state initial solution, the method reproduces the initial solution. In symbols it will be shown that

$$
\underline{\Psi}_{1}=E(h) \underline{\Psi}_{0}=\Psi_{0}
$$

for any thetas and any time step size. From Eq. (22) and the criticality condition $\mathrm{H}_{-0}=0$ we have

$$
\mathrm{R} \underline{\Psi}_{0}=-Q \underline{\Psi}_{0}
$$

so that Eq. (28) becomes

$$
\begin{aligned}
\underline{\Psi}_{1} & =[I-h \Theta R]^{-1}\left[e^{h Q}-h \Lambda Q-h \Theta R\right] \Psi_{0} \\
& =[I-h \Theta R]^{-1}[I-h \Theta R] \Psi_{0}=\Psi_{0},
\end{aligned}
$$

by use of Eq. (31)

\subsubsection{Asymptotic Behavior}

The solution methods under consideration will yield the exact asymptotic solution, when the corresponsing asymptotic thetas are used. We have, for an asymptotic reactor,

$$
\mathrm{H}_{0}=\omega_{0} \underline{\Psi}_{0}
$$

where $\omega_{0}$ is the asymptotic period. We have to show that 


$$
\underline{\Psi}_{1}=E(h) \underline{\Psi}_{0}=e^{h \omega_{0}} \underline{\Psi}_{0}
$$

for any $h$, and the proper $\theta$ matrix. From Eqs. (33) and (22) we can write

$$
\mathrm{R} \underline{\Psi}_{0}=\left[\omega_{0} I-Q\right] \underline{\Psi}_{0}
$$

Using this result and Eq. (31) in Eq. (28) we get

$$
\begin{aligned}
\underline{\Psi}_{1} & =[I-h \ominus R]^{-1}\left[e^{h Q}+h \Lambda\left(\omega_{0} I-Q\right)-h \ominus R\right] \underline{\Psi}_{0} \\
& =e^{h \omega 0_{\Psi_{0}}+[I-h \Theta R]^{-1}\left[h \omega_{0} \Lambda+\left(1-e^{h \omega} 0\right)\left\{I-h \Theta\left(\omega_{0} I-Q\right)\right\}\right] \underline{\Psi}_{0} .}
\end{aligned}
$$

Now, it is sufficient to show that

$$
\left[h \omega_{0} \Lambda+\left(I-e^{h \omega_{0}}\right)\left\{I-h \theta\left(\omega_{0} I-Q\right)\right\}\right]=0
$$

From Eqs. (20) and (25) we have for Method 1

$$
\theta=\left\{\frac{1}{h \omega_{0}}-\frac{1}{e^{h \omega_{0}}-1}\right\}
$$

for an asymptotic reactor, $Q=0$, and $\Lambda=I$. Equation (34) is readily seen to hold. For Method 2, from Eqs. (21) and (29) we get

$$
\left.\theta=\frac{1}{h \omega_{0}}\left[\frac{1}{h}\left(\omega_{0} I-Q\right)\right)^{-1}\left(e^{h \omega_{0}} I-e^{h Q}\right)-\Lambda\right]
$$

With this result and Eq. (31) we find that Eq. (34) is again true.

We can also show that, under certain conditions, Method 1 is asymptotically stable, meaning that the eigenvalue of 
largest magnitude of the advancement matrix $E(h)$ corresponds to the non-negative eigenvector of $\mathrm{H}$. This property can be demonstrated for the case of a scalar $\theta$ matrix, $\theta=\theta I$ in which case

$$
E(h)=[I-h \theta H]^{-1}[I+h(I-\theta) H]
$$

has the same eigenvectors as $H$. We assume further that the eigenvalues $\omega_{n}$ of $H$ are real and all $\omega_{n}<\omega_{0}$ where $\omega_{0}$ is the eigenvalue corresponding to the non-negative eigenvector of $H$. The eigenvalues of $E(h)$ are

$$
\nu_{n}=\frac{1+h(1-\theta) \omega_{n}}{1-h \theta \omega_{n}}
$$

We can infer from this expression that for a fixed $\theta$, we have $\left|\nu_{n}\right|<\nu_{0}$ if

$$
\frac{1}{2}\left[\frac{1}{\theta}-\frac{1}{1-\theta}\right] \leq h \omega_{0}<\frac{1}{\theta} .
$$

If we pick $\theta$ from Eq. (20) then $v_{0}=e^{h \omega_{0}}$ and the condition for asymptotic convergence becomes

$$
\left|\frac{1+h(1-\theta) \omega_{n}}{1-h \theta \omega_{n}}\right|<e^{h \omega_{0}}
$$

for $\omega_{n}<\omega_{0}$.

The condition (35) holds for any $\omega_{n}<\omega_{0}$ if $\omega_{0}>0$, but

fails for negative $\omega_{0}$ if $\omega_{n}$ is of very large magnitude.

\subsubsection{Solvability}

Solution of the time advancement equation (28) requires 
the existence of the inverse matrix in that equation. Equivalently, we must be able to calculate a unique $\Psi_{1}$ from the linear system of equations implied by Eq. (28) . A necessary and sufficient condition for the existence of this inverse is then that

$$
G=M_{1}-\frac{1}{h \theta_{0}} V^{-1}-\sum_{i=1}^{I} \beta_{i} \chi_{i} \frac{\theta_{i}}{\theta_{0}} F_{1}
$$

has an inverse, as can be concluded from the equivalent Eqs. (10). Equation (10-a) can be written as

$$
\mathrm{G}_{1}+\underline{s}=0
$$

which has the form of a steady-state equation with an external source. The operator $G$ corresponds to $M(t+h)$ given by Eq. (9), with an added absorption and a reduced delayed neutron production (since $\theta_{i} / \theta_{0}<1$, from Eq. (15)).

To find conditions under which $G$ has an inverse we use the properties of essentially positive matrices (43) which we now define: a real matrix $P$ is essentially positive if it is irreducible and has non-negative off-diagonal elements. Such a matrix has a real eigenvalue $\pi_{0}$ which is algebraically larger than $\operatorname{Re}\left(\pi_{i}\right)$ for any other eigenvalue $\pi_{i}$. To $\pi_{0}$ there corresponds a positive eigenvector which is the only non-negative eigenvector of the matrix. If any element of $P$ is increased $\pi_{0}$ also increases.

Let us now, consider the eigenvalue problem

$$
\mathrm{H}_{1} \underline{u}_{\mathrm{n}}=w_{\mathrm{n} \mathrm{u}_{\mathrm{n}}}
$$


The matrix $\mathrm{H}$ as defined after Eq. (5) is irreducible only if $\nu \Sigma_{f} \neq 0$ for all points. However, in case $\nu \Sigma_{f}=0$ for a particular region of the reactor, we can always redefine $H$ so as to make it always irreducible. This is done simply by deleting the $k^{\text {th }}$ column from the matrices $x_{i}$ and the $k^{\text {th }}$ row from the matrix $F$, whenever $\underline{f}_{k}=0$ (see sec. (3.2), for notation). Corresponding deletions are made in vectors on which $\mathrm{H}$ operates. Such a procedure has the effect of eliminating elements $c_{i k}$ from the vectors $C_{i}$, whenever $\underline{f}_{\mathrm{k}}=0$, and expresses the recognition that, physically, the precursor concentrations are zero for points at which no fission takes place. The above redefinition of $\mathrm{H}$ is tacitly assumed below.

An examination readily shows that $H$ is an essentially positive matrix. Let $\omega_{0}$ be its eigenvalue corresponding to $\underline{u}_{0}>0$, and let

$$
\underline{u}_{0}=\operatorname{col}\left[\underline{u}_{00} \underline{u}_{01} \underline{u}_{02} \cdots \underline{u}_{0 I}\right] .
$$

Solving Eq. (37) with $\mathrm{n}=0$ for $\underline{\mathrm{u}}_{0 i}$ in terms of $\underline{u}_{00}$ gives

$$
\underline{u}_{0 i}=\frac{\beta_{i}}{\omega_{0}+\lambda_{i}} F_{1} \underline{u}_{00}
$$

Since $\underline{u}_{0 i}$ and $\underline{u}_{00}$ have only positive elements, $F_{1}$ is a positive matrix, and $\beta_{i}>0$, we must necessarily have $\left(\omega_{0}+\lambda_{i}\right)$ $>0$, a result which will be implicitly assumed below. By using the expression above for $\underline{u}_{0 i}$ we can solve Eq. (37) 
with $\mathrm{n}=0$ for $\underline{u}_{0}$, obtaining

$$
G^{\prime} \underline{u}_{00}=\left[M_{1}-\omega_{0} v^{-1}-\sum_{i=1}^{I} \frac{\omega_{0}}{\omega_{0}+\lambda_{i}} \beta_{i} x_{i} F_{1}\right] \underline{u}_{00}=0
$$

Upon inspection it can be seen that $G^{\prime}$ is an essentially positive matrix with zero as the eigenvalue corresponding to the positive eigenvector $\underline{u}_{00}$. The matrix $G$ is also essentially positive. If

$$
\omega_{0} \leq \frac{1}{h \theta_{0}} \quad \text { and } \quad \frac{\omega_{0}}{\omega_{0}+\lambda_{i}} \leq \frac{\theta_{i}^{\prime}}{\theta_{0}} \quad(i=1,2, \ldots, I)
$$

with at least one strict inequality, G can be obtained from G' by decreasing some elements of the latter. From the properties of essentially positive matrices we can then conclude that, under the conditions (38) the real parts of all eigenvalues of $G$ are less than zero and thus $G$ will have $a$ negative inverse. (44) With that, a sufficient condition to get $\Psi_{1}>0$ is that $\underline{S}>0$, as can be gathered from Eqs. (36) and (10), by using Eq. (15) .

For Method 1, Eq. (38) reduces to $h \omega_{0} \theta_{i} \leq 1 \quad(i=0,1$, $2, \ldots$. I) with at least one strict inequality. It can be shown that the conditions (38) are always obeyed for both methods, if asymptotic thetas corresponding to $\omega_{0}$ (as given by Eqs. (20) and (21)) are used. This is easily seen to be the case, for Method 1. For Method 2, again the proofs are straightforward but somewhat more involved. 


\section{Chapter 4}

NUMERICAL RESULTS

\subsection{Results for Point Kinetics}

We now present some numerical results for two representative reactors, a thermal and a fast reactor. The values for $\Lambda, \beta_{i}$ and $\lambda_{i}$ (in $\sec ^{-1}$ ) for these reactors are shown in Table I, for six delayed neutron groups. We shall consider here five different transients, all starting from equilibrium conditions and with $\mathrm{n}(0)=1$. In every case the source term $f$ is taken to be zero. The Tables II to VI show the exact $n(t)$ and the relative percent errors of the calculations for several options of the method presented in this work, the errors being defined as

$$
\text { Error }=\frac{n_{\text {calc }}-n_{\text {exact }}}{n_{\text {exact }}} \times 100
$$

The results are shown for selected times $t$ during the transient and for several values of the time step size $h$ used in the calculations. The numbers in each square are in exponential notation and correspond, respectively, to the methods described in each of the cases that follow.

\section{Case 1}

This case corresponds to a step reactivity insertion of +0.5 dollars in the fast reactor, calculations having been done by three methods: (a) Padé $(2,0)$ with no explicit treatment of roots; (b) Padé $(1,1)$ with $\omega_{6}$ treated explicitly; 
TABLE I

Parameters for Two Typical Reactors

\begin{tabular}{|c|c|c|c|c|}
\hline \multirow{2}{*}{ NEUTRON } & \multicolumn{2}{|c|}{ THERMAL REACTOR } & \multicolumn{2}{c|}{ FAST REACTOR } \\
\cline { 2 - 5 } & $\lambda_{i}$ & $\beta_{i}$ & $\lambda_{i}$ & $\beta_{i}$ \\
\hline 1 & 0.0127 & $2.850 \times 10^{-4}$ & 0.0129 & $1.672 \times 10^{-4}$ \\
2 & 0.0317 & $1.5975 \times 10^{-3}$ & 0.0311 & $1.232 \times 10^{-3}$ \\
3 & 0.115 & $1.410 \times 10^{-3}$ & 0.134 & $9.504 \times 10^{-4}$ \\
4 & 0.311 & $3.0525 \times 10^{-3}$ & 0.331 & $1.443 \times 10^{-3}$ \\
5 & 1.40 & $9.600 \times 10^{-4}$ & 1.26 & $4.534 \times 10^{-4}$ \\
6 & 3.87 & $1.950 \times 10^{-4}$ & 3.21 & $1.540 \times 10^{-4}$ \\
\hline & $\beta_{\text {tot }}=0.00750$ & $\beta_{\text {tot }}=0.00440$ \\
$\Lambda$ & \multicolumn{2}{|c|}{$\Lambda=5 \times 10^{-4} \mathrm{sec}$} & $\Lambda=1 \times 10^{-7} \mathrm{sec}$ \\
\hline
\end{tabular}


(c) Crank-Nicholson. Results are presented in Table II. The errors for this case are of the same order of magnitude for the first two methods, (a) and (b), method (b) having an error usually about half of the error of method (a). The errors of the Crank-Nicholson method, (c), are in general unacceptably large, except for large $t$ and small $\mathrm{h}$. Comparison of the methods (b) and (c) shows the large correction effect obtained by treating $\omega_{6}$ explicitly, a feature to be shared by some of the following cases. We note that the errors for the first two methods are quite small (less than 18 ) for $h$ as large as 1 second.

\section{Case 2}

In this case, we perturb the thermal reactor by inserting a -0.5 dollars step reactivity. Calculations were done using the same three methods as for Case 1 . Table III shows the results.

For this case, we again have that the errors for the methods (a) and (b) are of the same order of magnitude, except for the early part of the transient ( $t \sim 0.1 \mathrm{sec}$ ) when the second method yields much smaller errors. The errors for method (a) are nevertheless also quite small. In this example, the Crank-Nicholson method, (c), fares better than for Case 1, but the errors become too large for time steps of 0.5 seconds or larger, in contrast with the first two methods. 
TABLE II

Percent Errors and Exact $n(t)$ for Case 1 .

\begin{tabular}{|c|c|c|c|c|}
\hline$h(\sec )$ & Method & $t=0.1 \mathrm{sec}$ & $t=1 \mathrm{sec}$ & $t=10 \mathrm{sec}$ \\
\hline 0.01 & $\begin{array}{l}a \\
b \\
c\end{array}$ & $\begin{array}{l}-9.64(-5) \\
+4.82(-5) \\
-4.02(+1)\end{array}$ & $\begin{array}{l}-7.53(-5) \\
+3.77(-5) \\
-6.11(+0)\end{array}$ & $\begin{array}{l}-7.85(-5) \\
0.0 \\
0.0\end{array}$ \\
\hline 0.10 & $\begin{array}{l}a \\
b \\
c\end{array}$ & $\begin{array}{l}-7.32(-3) \\
+4.53(-3) \\
+4.81(+1)\end{array}$ & $\begin{array}{l}-7.34 \cdot(-3) \\
+4.18(-3) \\
-3.70(+1)\end{array}$ & $\begin{array}{l}-6.28(-3) \\
+3.14(-3) \\
-6.54(+0)\end{array}$ \\
\hline 0.25 & $\begin{array}{l}a \\
b \\
c\end{array}$ & -- & $\begin{array}{l}-3.93(-2) \\
+2.62(-2) \\
-3.75(+1)\end{array}$ & $\begin{array}{l}-3.99(-2) \\
+1.94(-2) \\
-7.60(+0)\end{array}$ \\
\hline 0.50 & $\begin{array}{l}a \\
b \\
c\end{array}$ & -- & $\begin{array}{l}-1.29(-1) \\
+1.08(-1) \\
-3.75(+1)\end{array}$ & $\begin{array}{l}-1.64(-1) \\
+7.77(-2) \\
-7.71(+0)\end{array}$ \\
\hline 1.00 & $\begin{array}{l}a \\
b \\
c\end{array}$ & - & $\begin{array}{l}-3.91(-1) \\
+6.88(-1) \\
+3.83(+1)\end{array}$ & $\begin{array}{l}-6.93(-1) \\
+3.12(-1) \\
-7.52(+0)\end{array}$ \\
\hline \multicolumn{2}{|c|}{ Exact $n(t)$. } & 2.075317 & 2.655853 & 12.74654 \\
\hline
\end{tabular}

Method: (a) Padé $(2,0)$;

(b) Padé $(1,1)+\omega_{6}$ term;

(c) Crank-Nicholson. 
TABLE III

Percent Errors and Exact $n(t)$ for Case 2 .

\begin{tabular}{|c|c|c|c|c|}
\hline$h($ sec $)$ & Method & $t=0.1 \mathrm{sec}$ & $t=1 \mathrm{sec}$ & $t=10 \mathrm{sec}$ \\
\hline 0.01 & $\begin{array}{l}a \\
b \\
c\end{array}$ & $\begin{array}{l}+7.99(-2) \\
-2.86(-5) \\
-4.70(-2)\end{array}$ & $\begin{array}{l}+6.59(-5) \\
-4.94(-5) \\
-4.94(-5)\end{array}$ & $\begin{array}{l}0.0 \\
0.0 \\
0.0\end{array}$ \\
\hline 0.10 & $\begin{array}{l}a \\
b \\
c\end{array}$ & $\begin{array}{l}+3.15(+0) \\
-3.88(-3) \\
-7.78(+0)\end{array}$ & $\begin{array}{l}+6.44(-3) \\
-3.71(-3) \\
-3.71(-3)\end{array}$ & $\begin{array}{l}+7.07(-4) \\
-3.53(-4) \\
-3.53(-4)\end{array}$ \\
\hline 0.25 & $\begin{array}{l}a \\
b \\
c\end{array}$ & - & $\begin{array}{l}+3.44(-2) \\
-2.32(-2) \\
+2.83(+0)\end{array}$ & $\begin{array}{l}+4.37(-3) \\
-2.27(-3) \\
-2.27(-3)\end{array}$ \\
\hline 0.50 & $\begin{array}{l}a \\
b \\
c\end{array}$ & - & $\begin{array}{l}+1.19(-1) \\
-9.05(-2) \\
+2.62(+1)\end{array}$ & $\begin{array}{l}+1.69(-2) \\
-9.09(-3) \\
+5.92(-2)\end{array}$ \\
\hline 1.00 & $\begin{array}{l}\mathrm{a} \\
\mathrm{b} \\
\mathrm{c}\end{array}$ & -- & $\begin{array}{l}+5.01(-1) \\
-6.86(-1) \\
-4.55(+1)\end{array}$ & $\begin{array}{l}+6.34(-2) \\
-3.64(-2) \\
+1.41(+1)\end{array}$ \\
\hline Exac & & 0.6989252 & 0.6070536 & 0.3960777 \\
\hline
\end{tabular}

Method: (a) Padé $(2,0)$;

(b) Padé $(1,1)+w_{6}$ term;

(c) Crank-Nicholson. 
It is to be noted that the transient is very accurately represented in method (b) because of the explicit treatment of ${ }^{\omega}{ }_{6}$.

Again the errors with the first two methods are less than $1 \%$ even for time steps up to 1 second.

\section{Case 3}

This case is that of a step reactivity of +1 dollar introduced in the thermal reactor, four methods being used to do the calculations: (a) Padé $(2,0)$ with explicit treatment of $\omega_{0} ;(b)$ Padé $(2,0)$ without explicit treatment of roots; (c) Padé $(1,1)$ with $\omega_{0}$ treated explicitly; (d) Crank-Nicholson. Results are displayed in Table IV.

As one would expect, methods (a) and (c) have smaller errors than the other two, a situation which again shows the improvement obtained by the explicit treatment of the dominant root, in this case $\omega_{0}$. The errors are about the same for both methods, but method (c) fares better than method (a) for small h. A parallel behavior takes place for methods (b) and (d). Note that the errors for methods (a) and (c) are of the order of 18 for $h$ as large as 1 second.

\section{Case 4}

This case corresponds to a positive ramp insertion of reactivity of $+1 \mathrm{dollar} / \mathrm{sec}$ in the fast reactor. The calculations were done using two methods, namely: (a) Padé $(2,0)$ 
TABLE IV

Percent Errors and Exact $n(t)$ for Case 3 .

\begin{tabular}{|c|c|c|c|c|}
\hline$h($ sec) & Method & $t=0.1 \mathrm{sec}$ & $t=0.5 \mathrm{sec}$ & $t=1 \mathrm{sec}$ \\
\hline 0.01 & $\begin{array}{l}a \\
b \\
c \\
d\end{array}$ & $\begin{array}{l}-2.70(-3) \\
-5.76(-3) \\
+1.39(-3) \\
+2.90(-3)\end{array}$ & $\begin{array}{l}-7.72(-4) \\
-9.17(-3) \\
+4.83(-4) \\
+4.54(-3)\end{array}$ & $\begin{array}{l}-1.24(-4) \\
-1.50(-2) \\
+6.21(-5) \\
+7.40(-3)\end{array}$ \\
\hline 0.10 & $\begin{array}{l}a \\
b \\
c \\
d\end{array}$ & $\begin{array}{l}-2.16(-1) \\
-5.64(-1) \\
+1.43(-1) \\
+2.93(-1)\end{array}$ & $\begin{array}{l}-6.85(-2) \\
-1.02(+0) \\
+4.32(-2) \\
+4.57(-1)\end{array}$ & $\begin{array}{l}-1.03(-2) \\
-1.71(+0) \\
+6.21(-3) \\
+7.48(-1)\end{array}$ \\
\hline 0.25 & $\begin{array}{l}a \\
b \\
c \\
a\end{array}$ & $\quad--$ & $\begin{array}{l}-3.25(-1) \\
-7.53(+0) \\
+2.83(-1) \\
+2.98(+0)\end{array}$ & $\begin{array}{l}-5.22(-2) \\
-1.25(+1) \\
+3.89(-2) \\
+4.93(+0)\end{array}$ \\
\hline 0.50 & $\begin{array}{l}a \\
\therefore \quad b \\
\text { c } \\
\text { d }\end{array}$ & - & $\begin{array}{l}-9.04(-1) \\
-3.33(+1) \\
+1.46(+0) \\
+1.44(+1)\end{array}$ & $\begin{array}{l}-1.59(-1) \\
-4.99(+1) \\
+1.47(-1) \\
+2.46(+1)\end{array}$ \\
\hline 1.00 & $\begin{array}{l}a \\
b \\
c \\
d\end{array}$ & -- & - & $\begin{array}{r}-4.28(-1) \\
-9.08(+1) \\
+1.42(+0) \\
-1.32(+3)\end{array}$ \\
\hline \multicolumn{2}{|c|}{ Exact $n(t)$} & 2.515766 & 10.36253 & 32.18354 \\
\hline
\end{tabular}

Method: (a) Padé $(2,0)+\omega_{0}$ term;

(b) Padé $(2,0)$;

(c) Padé $(1,1)+w_{0}$ tèrm;

(d) Crank-Nicholson. 
with automatic inclusion of the $\omega_{0}$ term; (b) Padé $(2,0)$ with no explicit treatment of roots. By automatic inclusion of the $\omega_{0}$ term we mean that this root is treated explicitly whenever $h \omega_{0}$ is larger than a certain value, which we chose as 0.05 . Otherwise, the $\omega_{0}$ term is not included. Table $V$ shows the results for this case.

The results are exactly the same for $t=0.5 \mathrm{sec}$ and approximately the same for $t=1 \mathrm{sec}$, which indicates that the main source of error here is the non-constancy of the reactivity. The errors become very large in the vicinity of prompt-criticality (and beyond) for time steps of the order of 0.01 seconds or larger, especially for the results at $t=1$ sec. This behavior, however, represents no significant limitation to the method since, in most practical problems, feedback considerations will limit the acceptable time step size to a small value if results of a reasonable accuracy are desired.

\section{Case 5}

This case is that of a more complex reactivity insertion pattern in the thermal reactor: $a+1$ dollar/sec ramp up to 0.5 seconds followed by a -1 dollar/sec ramp up to 1 second; then a +1 dollar/sec ramp up to 1.5 seconds followed by a constant 0.5 dollars reactivity from then on. Note that $\rho(t)$ is a continuous function. Table VI shows the results obtained for this case by three methods: (a) Padé $(2,0)$ with no explicit treatment of roots; (b) Padé $(1,1)$ 
TABLE V

Percent Errors and Exact $n(t)$ for Case 4 .

\begin{tabular}{|c|c|c|c|}
\hline$h($ sec $)$ & Method & $t=0.5 \mathrm{sec}$ & $t=I \mathrm{sec}$ \\
\hline 0.001 & $\begin{array}{l}a \\
b\end{array}$ & $\begin{array}{l}-9.15(-2) \\
-9.15(-2)\end{array}$ & $\begin{array}{l}-5.07(-1) \\
-5.96(-1)\end{array}$ \\
\hline 0.01 & $\begin{array}{l}a \\
b\end{array}$ & $\begin{array}{l}-9.82(-1) \\
-9.82(-1)\end{array}$ & $\begin{array}{l}-2.92(+1) \\
-3.34(+1)\end{array}$ \\
\hline 0.10 & $\begin{array}{l}a \\
b\end{array}$ & $\begin{array}{l}-9.15(+0) \\
-9.15(+0)\end{array}$ & $\begin{array}{l}-9.48(+1) \\
-9.52(+1)\end{array}$ \\
\hline 0.25 & $\begin{array}{l}a \\
b\end{array}$ & $\begin{array}{l}-2.03(+1) \\
-2.03(+1)\end{array}$ & $\begin{array}{l}-9.85(+1) \\
-9.86(+1)\end{array}$ \\
\hline Exac & & 2.136407 & 1207.813 \\
\hline
\end{tabular}

Method: (a) Padé $(2,0)+\omega_{0}$ term $\left[h \omega_{0}>0.05\right]$;

(b) Padé $(2,0)$. 
TABLE VI

Percent Errors and Exact $n(t)$ for Case 5 .

\begin{tabular}{|c|c|c|c|c|c|c|}
\hline$h($ sec $)$ & Method & $t=0.5 \mathrm{sec}$ & $t=1 \mathrm{sec}$ & $t=1.5 \mathrm{sec}$ & $t=2 \mathrm{sec}$ & $t=10 \mathrm{sec}$ \\
\hline 0.01 & $\begin{array}{l}a \\
b \\
c\end{array}$ & $\begin{array}{l}-2.69(-2) \\
-4.24(-3) \\
-4.24(-3)\end{array}$ & $\begin{array}{l}+3.74(-2) \\
-3.30(-3) \\
-3.30(-3)\end{array}$ & $\begin{array}{l}-2.76(-2) \\
-4.76(-3) \\
-4.76(-3)\end{array}$ & $\begin{array}{l}-2.97(-3) \\
-4.36(-4) \\
-4.36(-4)\end{array}$ & $\begin{array}{l}-1.25(-3) \\
-5.81(-4) \\
-5.81(-4)\end{array}$ \\
\hline 0.10 & $\begin{array}{l}a \\
b \\
c\end{array}$ & $\begin{array}{l}-1.99(+0) \\
-4.22(-1) \\
-4.22(-1)\end{array}$ & $\begin{array}{l}+2.25(+0) \\
-3.50(-1) \\
-3.50(-1)\end{array}$ & $\begin{array}{l}-2.03(+0) \\
-4.70(-1) \\
-4.70(-1)\end{array}$ & $\begin{array}{l}-2.31(-1) \\
-4.28(-2) \\
-4.28(-2)\end{array}$ & $\begin{array}{l}-9.80(-2) \\
-5.10(-2) \\
-5.10(-2)\end{array}$ \\
\hline 0.25 & $\begin{array}{l}a \\
b \\
c\end{array}$ & $\begin{array}{l}-8.43(+0) \\
-6.64(+0) \\
-2.39(+0)\end{array}$ & $\begin{array}{l}+8.40(+0) \\
+6.07(+0) \\
-4.61(+0)\end{array}$ & $\begin{array}{l}-8.55(+0) \\
-6.94(+0) \\
-2.60(+0)\end{array}$ & $\begin{array}{l}-1.15(+0) \\
-6.42(-1) \\
-3.37(-1)\end{array}$ & $\begin{array}{l}-4.67(-1) \\
-3.85(-1) \\
-3.19(-1)\end{array}$ \\
\hline 0.50 & $\begin{array}{l}a \\
b \\
c\end{array}$ & $\begin{array}{l}-2.03(+1) \\
-1.94(+1) \\
-1.08(+1)\end{array}$ & $\begin{array}{l}+1.98(+1) \\
+2.00(+1) \\
+1.40(+1)\end{array}$ & $\begin{array}{l}-2.02(+1) \\
-2.01(+1) \\
-1.82(+1)\end{array}$ & $\begin{array}{l}-3.66(+0) \\
-1.77(+0) \\
+6.25(+0)\end{array}$ & $\begin{array}{l}-1.41(+0) \\
-1.21(+0) \\
-1.09(+0)\end{array}$ \\
\hline Exac & $n(t)$ & 1.721422 & 1.211127 & 1.892226 & 2.521601 & 12.04711 \\
\hline
\end{tabular}

Method: (a) Padé $(2,0)$;

(b) Padé $(1,1)+\omega_{6}$ term $\left[h \omega_{6}<\min \left(-2,-1.2 h \lambda_{6}\right)\right]$;

(c) Crank-Nicholson. 
with automatic inclusion of the $\omega_{6}$ term; (c) Crank-Nicholson. In the second method, (b), the $\omega_{6}$ term is included whenever $h \omega_{6}<\min \left(-2,-1,2 h \lambda_{6}\right)$.

The errors are, in general, the same for all three methods. For small $\mathrm{h}$ the results show a larger error for method (a) which should be expected from the theory and was noted in previous cases. From the methods (b) and (c) it can be concluded that the better results obtained for the Crank-Nicholson method are accidental, since method (b) is an improvement over Crank-Nicholson.

Notice also that the inaccurate results obtained for larger time steps stem from the very poor representation of the ramp as a series of steps. In particular, for $h=0.5$ sec, we simply have the transient represented as a 0.25 dollar step from 0 to 1.5 seconds followed by another step totalling 0.5 dollars from then on.

\subsection{Analysis of Point Kinetics Results}

The results of Sec. (4.1) for the first three cases show the great improvement of the Padé $(1,1)$ method with explicit treatment of $\omega_{0}$ or $\omega_{6}$ over the simple Crank-Nicholson method. This improvement is apparent not only in that greater accuracy is obtained for all time steps, but also in that larger time steps can be taken without incurring large errors.

The Padé $(2,0)$ method is also much better than the 
Crank-Nicholson method, for the cases of constant reactivi$t y$, as can be concluded from the results for the first three cases. Except for near or above prompt-criticality, the Pade $(2,0)$ method does quite well without the need for inclusion of the $\omega_{0}$ term and does not suffer from the oscillations present in the Crank-Nicholson method when $h$ is large. In general, the Pade $(1,1)$ method yields better results than the Pade $(2,0)$ method. A glance through Tables II to IV shows that halving the time step size $h$ for the first of these methods will produce better results than the second method with time step $h$. Note, however, that in many cases the Pade $(1,1)$ method will require one more root treated explicitly than will the Pade $(2,0)$ method. Also, such extra calculation will normally more than double the amount of computation per time step. It can thus be seen that the Pade $(2,0)$ method will be more economical, in general, even if the time step necessary to attain a given accuracy is smaller.

The results for the last two cases show that, for varying reactivity, inclusion of roots in the basic approximations does not make any significant improvement in accuracy. Calculations for other cases, in addition to those presented, have been done and confirmed the trends observed here which seem to hold in general and which agree with the theoretical expectations. 


\subsection{Results for Space-Dependent Kinetics}

Next, results are presented for two slab reactors without external source, one a thermal reactor, ${ }^{(45)}$ and the other a fast reactor. $(37,46)$ The two-group parameters for these reactors are presented in Tables VII and VIII, and their geometries are shown in Figs. 1 and 2. The reactors are made critical initially by dividing $\nu \Sigma_{f}$ by $k_{\text {eff }}$ Although these reactor configurations are by no means typical of real reactors, they are expected to display similar numerical properties. We shall consider several different transients all starting from equilibrium conditions. Except for the first test case, all transients are highly asymmetric.

For all cases in which point kinetics calculations were used to find the thetas, the method described in Appendix $B$ was applied, with inclusion of $\omega_{0}$ and $\omega_{I}$ terms in $\Sigma$ ' for $h^{\omega}{ }_{0}>0.05$ and $h^{\omega}{ }_{I}<\min \left(-2,-1.2 h \lambda_{I}\right)$, respectively. When point kinetics was not used in Method $1, \theta_{i}$ was always taken to be 0.5 ( $i=1$ to $I$ ) and $\theta_{0}$ chosen as described in each case. For Method 2, when point kinetics calculations were not used, $\theta(s)$ was taken to be either $s / h$ or 1 . For simplicity, these choices of $\theta(s)$ will be indicated by $\theta_{0}=0.5$ and $\theta_{0}=1$, respectively. For ramps, $\theta_{i}$ was chosen as 0.5 ( $i=0$ to $\left.I\right)$ in Method 1 , and $\theta(s)$ as $s / h$ in Method 2, for all cases. The reasons for these choices will be given later. The time step was doubled at the end of any ramp that was followed by a period during which reactor parameters remained constant. The motivation for choosing $\theta_{i}=0.5$ ( $i=0$ to $\left.I\right)$ or 
TABLE VII

Parameters for a Thermal Reactor

(all units in the CGS system) :

\begin{tabular}{|c|c|c|}
\hline Parameter & Regions $1 \& 3$ & Region 2 \\
\hline $\begin{array}{l}\mathrm{D}_{1} \\
\mathrm{D}_{2} \\
\Sigma_{\mathrm{r} 1} \\
\Sigma_{\mathrm{a} 2} \\
\Sigma_{\mathrm{s} 21} \\
\nu \Sigma_{f 1} \\
\nu \Sigma_{f 2}\end{array}$ & $\begin{array}{l}1.50 \\
0.50 \\
0.026 \\
0.180 \\
0.015 \\
0.010 \\
0.200\end{array}$ & $\begin{array}{l}1.00 \\
0.50 \\
0.020 \\
0.080 \\
0.010 \\
0.005 \\
0.099\end{array}$ \\
\hline $\begin{array}{l}x_{1}=1.0 \\
x_{2}=0.0\end{array}$ & $\begin{array}{l}v_{1}=1.0 \times 10^{7} \\
v_{2}=3.0 \times 10^{5}\end{array}$ & $\begin{array}{l}\Sigma_{\mathrm{I} 1}=\Sigma_{\mathrm{T} 1}-\Sigma_{\mathrm{s} 11} \\
\Sigma_{\mathrm{a} 2}=\Sigma_{\mathrm{T} 2}-\Sigma_{\mathrm{s} 22}\end{array}$ \\
\hline Group i & ${ }^{B_{i}}$ & $\lambda_{i}$ \\
\hline $\begin{array}{l}1 \\
2 \\
3 \\
4 \\
5 \\
6\end{array}$ & $\begin{array}{l}0.00025 \\
0.00164 \\
0.00147 \\
0.00296 \\
0.00086 \\
0.00032\end{array}$ & $\begin{array}{l}0.0124 \\
0.0305 \\
0.111 \\
0.301 \\
1.14 \\
3.01\end{array}$ \\
\hline $\begin{array}{l}\text { Regions } 1 \\
\text { Region } 2 \\
k_{\text {eff }}=0 . s\end{array}$ & $\begin{array}{l}\text { width }=40 \mathrm{~cm}, \\
\text { width }=160 \mathrm{~cm},\end{array}$ & $\begin{array}{l}\text { mesh size }=2.5 \mathrm{~cm} \\
\text { mesh size }=2.5 \mathrm{~cm}\end{array}$ \\
\hline
\end{tabular}


TABLE VIII

Parameters for a Fast Reactor

(all units in the CGS system).

\begin{tabular}{|c|c|c|}
\hline Parameter & Core & Blanket \\
\hline $\begin{array}{l}\mathrm{D}_{1} \\
\mathrm{D}_{2} \\
\Sigma_{\mathrm{r} 1} \\
\Sigma_{\mathrm{a} 2} \\
\Sigma_{\mathrm{s} 21} \\
v \Sigma_{\mathrm{fl}} \\
v \Sigma_{\mathrm{f} 2}\end{array}$ & $\begin{array}{l}2.0770 \\
0.9322 \\
0.020010 \\
0.015030 \\
0.014730 \\
0.008916 \\
0.014230\end{array}$ & $\begin{array}{l}1.5480 \\
0.9484 \\
0.016137 \\
0.008703 \\
0.013070 \\
0.000216 \\
0.000446\end{array}$ \\
\hline $\begin{array}{l}x_{1}=1.0 \\
x_{2}=0.0\end{array}$ & $\begin{array}{l}v_{1}=1.12 \times 10^{10} \\
v_{2}=1.18 \times 10^{9}\end{array}$ & $\begin{array}{l}=\Sigma_{\mathrm{T} 1}-\Sigma_{\mathrm{S} 11} \\
=\Sigma_{\mathrm{T} 2}-\Sigma_{\mathrm{s} 22}\end{array}$ \\
\hline$I=1$ & $\beta=0.003$ & $\lambda=0.065$ \\
\hline $\begin{array}{l}\text { Core: } \\
\text { Blanket: } \\
k_{\text {eff }}=0 .\end{array}$ & $\begin{array}{l}\text { width }=70 \mathrm{~cm} \\
\text { width }=48 \mathrm{~cm} \\
9\end{array}$ & $\begin{array}{l}\text { h size }=5 \mathrm{~cm} \\
\text { h size }=8 \mathrm{~cm}\end{array}$ \\
\hline
\end{tabular}




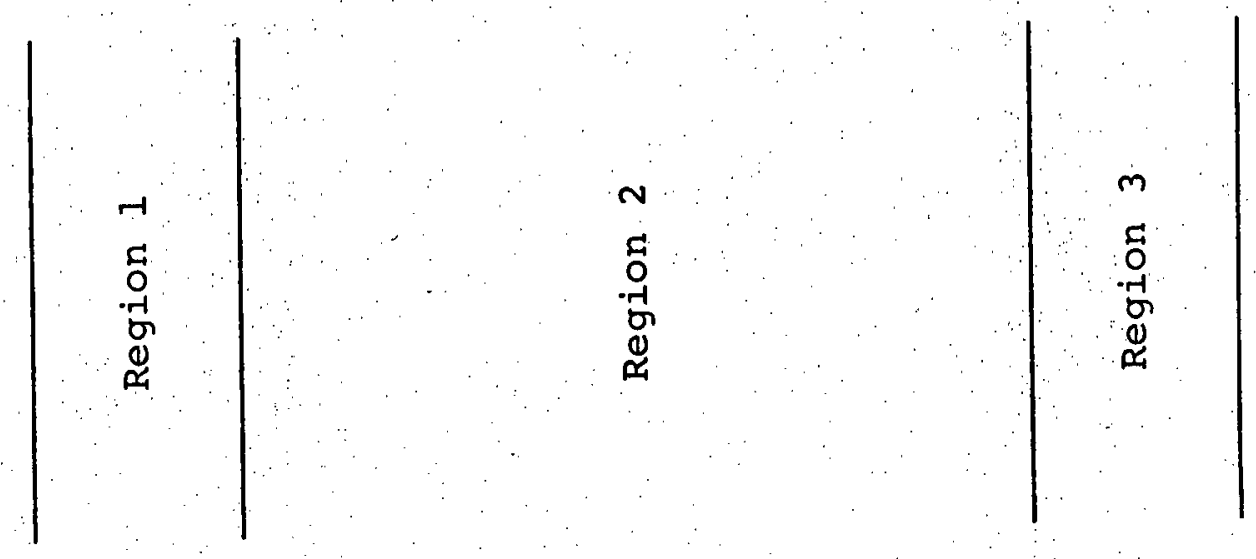

Fig. 1. Geometry for a Thermal Reactor.

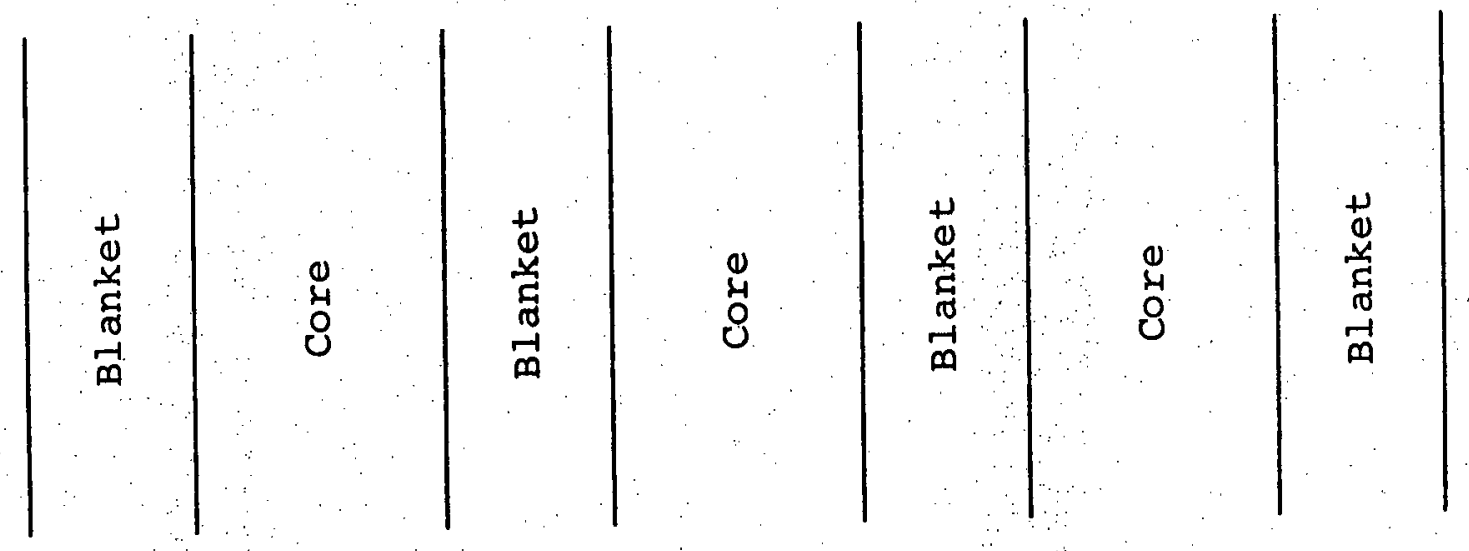

Fig. 2. Geometry for a Fast Reactor. 
$\theta(s)=s / h$ lies in the fact that, as seen in sec. (3.6), they yield a $O\left(\mathrm{~h}^{2}\right)$ global accuracy. Other choices will be accurate only to $O(h)$, globally. In practice, these choices of thetas usually yields the best results, if fixed thetas are to be used, except after steps or steep ramps in prompt-subcritical reactors. In such cases, use of $\theta_{0}=1$. or $\theta(s)=1$ will rapidly attenuate modes with large negative time constants which would otherwise make the solution oscillatory.

Case A

In this first case $\nu \Sigma_{f}$ was increased everywhere by $0.4 \%$ in a step fashion, in the thermal reactor. About $0.5 \mathrm{dol}$ lars of reactivity was thereby added. Several methods were used to perform the calculations. Some of the results are presented in Table IX, which shows for several time step sizes $h$, the fast flux in the midale of Region 1 or 3, at $t=0.5 \mathrm{sec}$ and $t=5 \mathrm{sec}$.

To obtain column A, Method 1 was used with thetas calculated from point kinetics. Method 2 was also applied under the same conditions, and led to essentially the same results. Column $B$ shows the same calculation as in column $A$ but with $\theta_{0}$ taken as 1 for the first step. In $\operatorname{column} C$ we used Method 2 with $\theta_{0}=1$ for the first two steps and $\theta_{0}=0.5$ from then on. Column $D$ shows the results using Method 1 with $\theta_{0}=1$, for the first two steps, and $\theta_{0}=0.5$ thereafter. 
TABLE IX

Flux Values for Case $A$.

\begin{tabular}{|c|c|c|c|c|c|c|c|c|c|c|}
\hline \multirow[t]{2}{*}{$\begin{array}{c}\mathrm{h} \\
(\mathrm{sec})\end{array}$} & \multicolumn{2}{|c|}{$\begin{array}{c}(A) \\
\text { Method } 1 \\
(\theta=\text { p.k. })+\end{array}$} & \multicolumn{2}{|c|}{$\begin{array}{c}\text { (B) } \\
\text { Method })^{*} \\
(\theta=\mathrm{p}, \mathrm{k} .)^{+}\end{array}$} & \multicolumn{2}{|c|}{$\begin{array}{l}\text { (c) } \\
\text { Method } 2 * * \\
\left(\theta_{0}=0.5\right)\end{array}$} & \multicolumn{2}{|c|}{$\begin{array}{c}(D) \\
\text { Method } 1 * * \\
\left(\theta_{0}=0.5\right)\end{array}$} & \multicolumn{2}{|c|}{$\begin{array}{c}(E) \\
\text { Method } 1 \\
\left(\theta_{0}=1\right)\end{array}$} \\
\hline & $.5 \mathrm{sec}$ & 5 sec & $.5 \mathrm{sec}$ & $5 \mathrm{sec}$ & $.5 \mathrm{sec}$ & $5 \mathrm{sec}$ & $.5 \mathrm{sec}$ & $5 \mathrm{sec}$ & $.5 \mathrm{sec}$ & $5 \mathrm{sec}$ \\
\hline 1.00 & - & 7.5669 & - & 7.5699 & - & 7.7400 & - & 7.9602 & - & 6.9793 \\
\hline 0.50 & 3.1673 & 7.5658 & 2.9281 & 7.5667 & 2.9445 & 7.5142 & 2.9281 & 7.4253 & 2.9281 & 7.2591 \\
\hline 0.25 & 3.1618 & 7.5664 & 3.1620 & 7.5668 & 3.0562 & 7.5578 & 3.0532 & 7.5429 & 3.0532 & 7.4090 \\
\hline 0.10 & 3.1622 & 7.5654 & 3.1612 & 7.5660 & 3.1848 & 7.5653 & 3.1873 & 7.5655 & 3.1186 & 7.5023 \\
\hline 0.05 & 3.1589 & 7.5652 & 3.1608 & 7.5659 & 3.1596 & 7.5658 & 3.1595 & 7.5658 & 3.1398 & 7.5339 \\
\hline $\begin{array}{l}\text { EXACT } \\
\text { FUUX }\end{array}$ & & .01 & 7 & & $\phi(0.5)$ & 3 & & $0)$ & 7.56 & \\
\hline
\end{tabular}

* $\theta_{0}=1$ for first step

+ p.k. = point kinetics thetas

** $\theta_{0}=1$ for first two steps 
This last calculation was repeated taking $\theta_{0}=1$ all the way, results being presented in column $\mathrm{E}$.

Not shown in the table are calculations done with the Crank-Nicholson method $\left(\theta_{0}=0.5\right)$ and with Method 2 taking $\theta_{0}=0.5$, as well as calculations analogous to those of columns $C$ and $D$ but with $\theta_{0}=1$ for only the first step. The Crank-Nicholson results and their counterparts for Method 2 were oscillatory, as expected from theory, yielding large errors. The last two calculations tended to be slightly less accurate than their counterparts, in C and D.

Examining Table IX we see that column A displays the most accurate results, except when $h$ is quite small. This behavior is probably due to some very small oscillations in the calculated thetas. The solution itself oscillates slightly, but oscillations are small enough that the first difference does not change sign. The theta oscillations are greatly reduced by the procedure described above leading to column B. Taking $\theta_{0}=1$ for the first time step makes the errors decrease for small $h$ but increase for large $h$ as can be seen by comparison of columns $A$ and $B$.

In spite of these anomalies, the results in column $A$ are very good. This is no surprise, of course, since in this case the assumption of constant flux shape is valid. Comparison of columns C and D shows that Method $I$ is slightly better than Method 2 for this choice of thetas. The procedure used in TWIGL of choosing $\theta_{0}=1$ all the way is clearly the worse, except for the first two time steps when col- 
umns $D$ and $E$ coincide.

\section{Case B}

A transient was induced in the thermal reactor by a step reduction of $\Sigma_{a 2}$ in Region 1 by 18 . This is a transient in the delayed supercritical range. Results using various methods are presented in Table $\mathrm{X}$ which shows, for several values of $h$, the fast flux in the middle of Regions 1 and 3 for $t=0.5 \mathrm{sec}$ and $t=5 \mathrm{sec}$.

Columns $A$ and $B$ correspond to calculations with Method 2 and Method 1, respectively, with $\theta_{0}=1$ for the first time step, and thetas calculated from point kinetics for subsequent time steps. Columns $C$ and $D$ display similar results by Method 2 and Method 1 but with $\theta_{0}=0.5$ after the first time step. In column $\mathrm{E}$ we took $\theta_{0}=1$ for all time steps and used Method 1.

Calculations corresponding to columns $A$ and $B$ were also done without taking $\theta_{0}=1$ for the first step. While the calculated value for the first step was much better, all subsequent steps had inferior results. Also oscillations of the theta values took place; these were not present in the calculations for columns A and B. Such behavior is understandable, in view of the large change in shape that takes place in the first time step due to the fast modes (prompt jump). The point kinetics estimation of thetas for this time step is thus of little value. 
TABLE $X$

Flux Values for Case B.

\begin{tabular}{|c|c|c|c|c|c|c|c|c|c|c|c|}
\hline \multirow{2}{*}{ (sec) } & \multirow{2}{*}{ 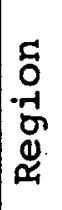 } & \multicolumn{2}{|c|}{$\begin{array}{c}\text { (A) } \\
\text { Method } 2^{*} \\
(\theta=\text { p.k. })+\end{array}$} & \multicolumn{2}{|c|}{$\begin{array}{l}\text { (B) } \\
\operatorname{Method~} 1^{*} \\
(\theta=\text { p.k. })+\end{array}$} & \multicolumn{2}{|c|}{$\begin{array}{c}(c) \\
\text { Method } 2^{*} \\
\left(\theta_{0}=0.5\right)\end{array}$} & \multicolumn{2}{|c|}{$\begin{array}{c}\text { (D) } \\
\text { Method } I^{*} \\
\left(\theta_{0}=0.5\right)\end{array}$} & \multicolumn{2}{|c|}{$\begin{array}{c}\text { (E) } \\
\text { Method } 1 \\
\left(\theta_{0}=1.0\right)\end{array}$} \\
\hline & & $.5 \mathrm{sec}$ & $5 \mathrm{sec}$ & $.5 \mathrm{sec}$ & $5 \mathrm{sec}$ & $.5 \mathrm{sec}$ & $5 \mathrm{sec}$ & $.5 \mathrm{sec}$ & $5 \mathrm{sec}$ & $.5 \mathrm{sec}$ & $5 \mathrm{sec}$ \\
\hline \multirow{2}{*}{1.00} & 1 & - & 9.5801 & - & 9.5858 & - & 9.3946 & - & 9.0746 & - & 8.7727 \\
\hline & 3 & - & 2.3549 & - & 2.3568 & - & 2.3364 & - & 2.2981 & - & 2.2454 \\
\hline \multirow{2}{*}{0.50} & 1 & 3.3579 & 9.5795 & 3.3366 & 9.5836 & 3.3579 & 9.6354 & 3.3366 & 9.7942 & 3.3366 & 9.1555 \\
\hline & 3 & 1.5607 & 2.3527 & 1.5583 & 2.3529 & 1.5607 & 2.3562 & 1.5583 & 2.3747 & 1.5583 & 2.2963 \\
\hline \multirow{2}{*}{0.25} & 1 & 3.6310 & 9.5800 & 3.6333 & 9.5809 & 3.7867 & 9.5891 & 3.8036 & 9.6177 & 3.4972 & 9.3622 \\
\hline & 3 & 1.5903 & 2.3532 & 1.5906 & 2.3533 & 1.6071 & 2.3527 & 1.6092 & 2.3551 & 1.5757 & 2.3240 \\
\hline \multirow{2}{*}{0.10} & 1 & 3.6352 & 9.5793 & 3.6355 & 9.5795 & 3.5845 & 9.5803 & 3.5783 & 9.5809 & 3.5815 & 9.4914 \\
\hline & 3 & 1.5914 & 2.3532 & 1.5914 & 2.3532 & 1.5874 & 2.3533 & 1.5867 & 2.3534 & 1.5852 & 2.3414 \\
\hline \multirow{2}{*}{0.05} & 1 & 3.6355 & 9.5794 & $3: 6356$ & 9.5794 & 3.6399 & 9.5800 & 3.6404 & 9.5802 & 3.6090 & 9.5354 \\
\hline & 3 & 1.5912 & 2.3532 & 1.5912 & 2.3532 & 1.5907 & 2.3533 & 1.5906 & 2.3533 & 1.5883 & 2.3473 \\
\hline EXACT & 1 & \multirow{2}{*}{\multicolumn{2}{|c|}{$\phi(0.0)$}} & $=1.4202$ & & \multirow[t]{2}{*}{$\phi(0.5)$} & $=3.636$ & & $\phi(5.0)$ & \multicolumn{2}{|l|}{$=9.5799$} \\
\hline FLUX & 3 & & & \multicolumn{2}{|c|}{$=1.4202 \%$} & & \multicolumn{3}{|c|}{$=1.5914}$, & \multicolumn{2}{|c|}{$=2.3533$} \\
\hline
\end{tabular}

* $\theta_{0}=1$ for first step

$+\mathrm{p} . \mathrm{k} .=$ point kinetics thetas 
Calculations using the Crank-Nicholson method and its equivalent for Method 2 were also performed. Solutions had large errors and were oscillatory in nature.

Finally, columns $D$ and $E$ were recalculated using $\theta_{0}=1$ for the first two steps. The accuracy of the results was similar to that shown for columns $D$ and $E$ in Table $X$.

Columns $A$ and $B$ of Table $X$ are the most accurate results for the larger time step sizes, with column A only slightly better, overall. Thus the two methods have about the same accuracy, for thetas calculated from point kinetics. Column $C$, however, displays generally better results

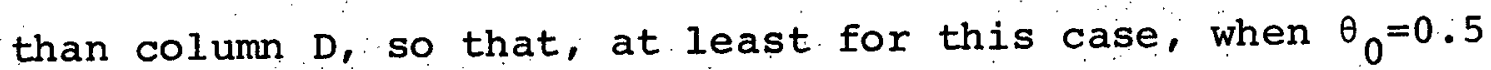
is used, Method 2 fares better. Comparing columns $D$ and $E$, one again sees the great improvement obtained by using $\theta_{0}=1$. only at the first one or two steps.

\section{Case C}

This case involves a step reduction of $\Sigma_{a 2}$ in Region 1 of the thermal reactor by 5\%. Such a perturbation puts the reactor in a prompt-critical state. Table XI shows the fast flux at $t=0.001$ and 0.005 sec in the middle of Regions 1 and 3 , calculated for several values of the time step $h$. The results were obtained by two methods, the CrankNicholson method, and Method 1 with thetas calculated from point kinetics. The corresponding results obtained from Method 2, with $\theta_{0}=0.5$ and point kinetics thetas, differ from the Method 1 results by at most one unit in the last digit. 
TABIE XI

Flux Values for Case $C$.

\begin{tabular}{|c|c|c|c|c|c|}
\hline \multirow{2}{*}{$\begin{array}{c}\mathrm{h} \\
(\mathrm{sec})\end{array}$} & \multirow{2}{*}{$\begin{array}{l}5 \\
0 \\
0 \\
0 \\
ه ్ 4\end{array}$} & \multicolumn{2}{|c|}{ CRANK-NICHOLSON } & \multicolumn{2}{|c|}{ THETA METHOD } \\
\hline & & $0.001 \mathrm{sec}$ & $0.005 \mathrm{sec}$ & $0.001 \mathrm{sec}$ & $0.005 \mathrm{sec}$ \\
\hline \multirow{2}{*}{$1 \times 10^{-3}$} & 1 & 4.5981 & 130.06 & 4.5611 & 112.79 \\
\hline & 3 & 1.4242 & 2.4403 & 1.4239 & 2.2887 \\
\hline \multirow{2}{*}{$5 \times 10^{-4}$} & 1 & 4.3711 & 111.49 & 4.3552 & 107.68 \\
\hline & 3 & 1.4220 & 2.2782 & 1.4219 & 2.2445 \\
\hline \multirow{2}{*}{$2.5 \times 10^{-4}$} & 1 & 4.3448 & 107.61 & 4.3417 & 106.68 \\
\hline & 3 & 1.4214 & 2.2444 & 1.4214 & 2.2362 \\
\hline \multirow{2}{*}{$1 \times 10^{-4}$} & 1 & 4.3274 & 106.57 & 4.3274 & 106.44 \\
\hline & 3 & 1.4212 & 2.2354 & 1.4212 & 2.2342 \\
\hline \multirow{2}{*}{$5 \times 10^{-5}$} & 1 & 4.3236 & 106.42 & 4.3236 & 106.42 \\
\hline & 3 & 1.4212 & 2.2341 & 1.4212 & 2.2341 \\
\hline EXACT & 1 & \multicolumn{4}{|c|}{$\phi(0)=1.4202, \phi(0.001)=4.3231, \phi=(0.005)=106.37$} \\
\hline FLUX & 3 & $=1.420$ & & 212 ; & $=2.2337$ \\
\hline
\end{tabular}


This behavior was expected because of the small $h$ required for the calculation of a prompt-supercritical transient. Here we have a case in which the fundamental mode is strongly dominant, and the point kinetics thetas produce sizable improvements in accuracy. This improvement is particularly evident for the values at $t=0.005 \mathrm{sec}$. The values for $t=0.001$ sec with $h=10^{-4} \mathrm{sec}$, and all values for $h=5 \times 10^{-5}$ sec coincide for both methods because, as stated previously, the $\omega_{0}$ terms were included in the point kinetics calculation only for $h \omega_{0}>0.05$. Continued inclusion of such terms would have kept the superiority of the theta method over the Crank-Nicholson method. It can be seen from Table XI that, for roughly comparable accuracy, it is necessary to use for the Crank-Nicholson method time steps about half as large as those used with the theta method.

Case D

In this case a transient is provoked by reducing the absorption cross sections linearly in the right core of the fast reactor by $0.5 \%$ during the time interval from $t=0$ to $t=0.5 \mathrm{sec}$, and holding these values constant thereafter. The result is a delayed supercritical transient. Table XII shows, for several values of $h$, the fast flux at $t=0.5$ sec for the middle points of the outer cores, for Methods 1 and 2 using $\theta_{0}=0.5$

For Method 1 this amounts to a modified Crank-Nicholson scheme given by 
TABLE XII

Flux Values for Case D.

\begin{tabular}{|c|c|c|c|}
\hline $\begin{array}{c}h \\
(s e c)\end{array}$ & Core & Method 1 & Method 2 \\
\hline 0.50 & $\begin{array}{l}\text { Left } \\
\text { Right }\end{array}$ & $\begin{array}{l}1.7667 \\
7.231 .8\end{array}$ & $\begin{array}{l}1.7481 \\
7.0902\end{array}$ \\
\hline 0.25 & $\begin{array}{l}\text { Left } \\
\text { Right }\end{array}$ & $\begin{array}{l}1.7465 \\
7.0773\end{array}$ & $\begin{array}{l}1.7400 \\
7.0279\end{array}$ \\
\hline 0.10 & $\begin{array}{l}\text { Left } \\
\text { Right }\end{array}$ & $\begin{array}{l}1.7383 \\
7.0149\end{array}$ & $\begin{array}{r}1.7366 \\
7.0019\end{array}$ \\
\hline 0.05 & $\begin{array}{l}\text { Left } \\
\text { Right }\end{array}$ & $\begin{array}{l}1.7368 \\
7.0038\end{array}$ & $\begin{array}{l}1.7364 \\
7.0002\end{array}$ \\
\hline 0.025 & $\begin{array}{l}\text { Left } \\
\text { Right }\end{array}$ & $\begin{array}{l}1.7364 \\
7.0006\end{array}$ & $\begin{array}{r}1.7363 \\
6.9997\end{array}$ \\
\hline $\begin{array}{l}\text { EXACT } \\
\text { FLUX }\end{array}$ & $\begin{array}{l}\text { Left } \\
\text { Right }\end{array}$ & $\begin{aligned} \phi(0.0) & =1.1443 \\
& =1.1443\end{aligned}$ & $\begin{aligned} \phi(0.5) & =1.7363 \\
& =6.9995\end{aligned}$ \\
\hline
\end{tabular}




$$
\Psi_{1}=\left[I-\frac{1}{2} \mathrm{hH}_{1}\right]^{-1}\left[I+\frac{1}{2} \mathrm{hH}_{0}\right] \Psi_{0}
$$

It is seen from Table XII that the results using Method 2 are much better, in that the errors are two to three times smaller for the same $h$, or in that one must roughly halve the time step in Method 1 to match the accuracy of Method 2 .

Methods 1 and 2 with $\theta_{0}=0.5$ are of comparable accuracy in the constant parameter part of the transient, after $t=0.5$ sec. For this part we also used point kinetics thetas, in which case Method 2 fared slightly better than Method 1 . Comparing values obtained for this case using point kinetics thetas and $\theta_{0}=0.5$, one concludes that, for both methods, the $\theta_{0}=0.5$ choice is generally better for smaller $h$, the converse being true for larger $h$.

Performing the same calculation as in Ref. (37) with Method 1 shows that use of $\theta_{0}=0.5$ yields better results than the ones shown in that reference, using the thetas described there.

A similar computation was performed for the thermal reactor in which we linearly reduced $\Sigma_{a 2}$ in Region 1 by $1 \%$ in 1 sec, and held the parameters constant from then on. The results at the end of the ramp section, at $t=1$ sec, yielded the same conclusion about the higher accuracy of Method 2 over Method I, with $\theta_{0}=0.5$. For the constant parameter part of the transient, after $t=1$ sec, we found that again Methods 1 and 2 produced results of comparable accuracy. When point 
kinetics thetas were used, Method 2 was found again slightly better than Method 1 , which suffered from oscillation in its thetas, for the larger $h$. At the early part of the constant section ( $\operatorname{t} 2 \mathrm{sec})$, the use of point kinetics thetas improved results. However, later in time (tน5 sec), values obtained with $\theta_{0}=0.5$ all the way were more accurate.

Case E

In this case we introduce a subcritical transient in the thermal reactor by increasing $\Sigma_{a 2}$ linearly in Region 1 by $3 \%$ from $t=0$ to $t=1$ sec, and keeping parameters constant thereafter. In Table XIII we show, for several $h$, the values of the fast flux in the middle point of Regions 1 and 3 at $t=1 \mathrm{sec}$, using Methods 1 and 2 with $\theta_{0}=0.5$.

It can be seen from Table XIII that with Method 1 , the modified Crank-Nicholson method, results are better than with Method 2, except for the smaller $h$ when they both tend to agree, as expected. Results for the constant reactivity part of the transient, after $t=1 \mathrm{sec}$, are not shown here. They indicate however, that the Methods 1 and 2 give overall results of comparable accuracy, either using $\theta_{0}=0.5$ or thetas from point kinetics.

Use of point kinetics thetas again improved the results early in the constant parameter portion of the transient but, as time increases and $h$ decreases, they tend to have a lower accuracy in comparison with the $\theta_{0}=0.5$ results. The thetas 
TABLE XIII

Flux Values for Case E.

\begin{tabular}{|c|c|c|c|}
\hline $\begin{array}{c}\mathrm{h} \\
(\mathrm{sec})\end{array}$ & Region & Method 1 & Method 2 \\
\hline 0.50 & $\begin{array}{l}1 \\
3\end{array}$ & $\begin{array}{l}0.49608 \\
1.3451\end{array}$ & $\begin{array}{c}0.49077 \\
1.3438\end{array}$ \\
\hline 0.25 & $\begin{array}{l}1 \\
3\end{array}$ & $\begin{array}{l}0.49339 \\
1.3443\end{array}$ & $\begin{array}{l}0.49208 \\
1.3440\end{array}$ \\
\hline 0.10 & $\begin{array}{l}1 \\
3\end{array}$ & $\begin{array}{l}0.49335 \\
1.3444\end{array}$ & $\begin{array}{l}0.49328 \\
1.3444\end{array}$ \\
\hline 0.05 & $\begin{array}{l}1 . \\
3\end{array}$ & $\begin{array}{l}0.49418 \\
1.3445\end{array}$ & $\begin{array}{l}0.49418 \\
1.3445\end{array}$ \\
\hline 0.025 & $\begin{array}{l}1 \\
3\end{array}$ & $\begin{array}{l}0.49433 \\
1.3445\end{array}$ & $\begin{array}{l}0.49432 \\
1.3445\end{array}$ \\
\hline $\begin{array}{l}\text { EXACT } \\
\text { FLUX }\end{array}$ & $\begin{array}{l}1 \\
3\end{array}$ & $\begin{aligned} \phi(0.0) & =1.4202, \\
& =1.4202,\end{aligned}$ & $\begin{aligned} \phi(0.5) & =0.49432 \\
& =1.3445\end{aligned}$ \\
\hline
\end{tabular}


tend to oscillate after a while, the oscillations becoming larger as $h$ decreases, even yielding thetas outside the range $(0,1)$. The computer program used in the calculation limits them to $0.1<\theta \leq 1$. Those thetas oscillations probably account for the better accuracy of the calculations with a fixed $\theta_{0}$ of 0.5 for small $h$. Use of $\theta_{0}=1$ for the first step after $t=1$ sec would probably eliminate the theta oscillations and yield better results using point kinetics thetas for the subsequent time steps, as was the case in the step problems below prompt-critical analyzed before.

An analogous calculation was done for the fast reactor, by linearly increasing the absorption cross sections of the right core by 0.58 in 0.5 sec, and then holding it constant. The value found at $t=0.5 \mathrm{sec}$ again shows Method 1 to be more accurate than Method 2 , with $\theta_{0}=0.5$. Calculations using both methods and either $\theta_{0}=0.5$ or point kinetics thetas were all of similar accuracy for the flat portion of the transient.

It should be noted here that the conclusion about the accuracy of Methods 1 and 2, for this subcritical ramp, is the reverse of the one reached in the previous case of the delayed supercritical ramp, for both the thermal and the fast reactor.

\subsection{Analysis of Space Kinetics Results}

From the results of sec. (4.2) it is hard to see any general trend which would permit us to draw conclusions 
about which method is better. We saw in Case $A$ that, for the supposedly ideal case of constant shape, some form of weak instability is reflected in the oscillations found in the computed theta values. While this did not prove to be a serious problem it is certainly an unpleasant fact, and we found it useful to deal with it by the special procedure of taking $\theta_{0}=1$ for the first one or two steps.

When there is non-uniform reactivity insertion below. prompt-critical, either in step problems or in the constant reactivity part after initial ramps, the use of point kinetics thetas aid improve the results for the larger values of $h$ as can be seen from the results for Cases $B, D, E$ and $F$. The additional computing time to find these parameters can, however, be appreciable, and might be better put to use by taking a smaller time step especially since, in some cases the results based on computed thetas are less accurate then those using $\theta_{0}=0.5$. The above argument is even stronger for ramp insertions and for this reason (as discussed in sec. (4.3)) it was decided to abandon the point kinetics thetas for ramps in all cases calculated.

With respect to the relative merits of Methods 1 and 2 we found that, in delayed supercritical problems (Case D), Method 2 produced better results for the ramp section of the transient, for both the fast and the thermal reactor. That would be a result of general interest if it were not for the fact that the conclusion is reversed for the subcritical problems considered in case $E$. It may be, however, that the 
contrary behavior observed holds in general for the two separate classes of problems. We did not investigate this possibility in sufficient depth to justify such a general conclusion, however.

In prompt-supercritical problems, because of the necessarily small time steps, Methods 1 and 2 essentially agree. It was found, in Case $c$, that point kinetics thetas did produce a significant improvement in accuracy. This is a case in which a single mode is strongly dominant. In these cases one might try to find the asymptotic period by some simpler method and thus avoid the penalty imposed by the point kinetics related calculations.

\subsection{Additional Results for Space-Dependent Kinetics}

We quote here other interesting results of our study in which we compare the chosen methods with other variations. We shall use the fast reactor with the same ramp as that described in Case D.

First we compare Method 2 using $\theta(s)=s / h$ (that is assuming $L \Phi$ and $F \Phi$ to vary linearly between their initial and final values) with another calculation using Eq. (3.12) in which $L(t)$ and $F(t)$ are given their real values and only $\Phi(t)$ is assumed to vary linearly. The latter is equivalent to a linear spline approximation for the flux.

The errors for the fast flux in the midale points of the three cores (left, middle, and right, respectively) for 
the two methods are shown in Table XIV, for $t=0.5 \mathrm{sec}$. They indicate that the linear spline method is far less accurate. In trying to understand the reason for this, we consider a linear $L(t)$ and $F(t)$ and take a linear flux approximation. This gives, for instance,

$$
\frac{1}{h} \int_{0}^{h} M(t+s) \Phi(t+s) d s=\frac{1}{2}\left[\left(M_{0}+\frac{1}{3} \Delta M\right) \Phi_{0}+\left(M_{0}+\frac{2}{3} \Delta M\right) \Phi_{1}\right]
$$

where $\Delta M=M_{1}-M_{0} \cdot$ Thus, the operator at one third of the interval operates on the initial flux, and the operator at two thirds of the interval operates on its final value. In the method we adopted one would have

$$
\frac{1}{h} \int_{0}^{h} M(t+s) \Phi(t+s) d s=\frac{1}{2}\left[M_{0} \Phi_{0}+M_{1} \Phi_{1}\right]
$$

so that the operator at a given time $t$ acts on the flux for the same time $t$. This fact, we conjecture, accounts for the greater accuracy of Method 2 as compared to a linear spline approximation, at least for cases below prompt-critical.

Another variation studied was to assume the varying properties to be replaced by their average values during the time step. It was also found to be much less accurate than Methods 1 or 2 with $\theta_{0}=0.5$. For the case of a ramp, this amounts to substituting for the ramp a series of steps with the result that the global error, for time steps large in comparison with the prompt mode time constant, becomes $0(\mathrm{~h})$ instead of $O\left(\mathrm{~h}^{2}\right)$ as shown in Sec. (2.4). The flux tends to go through a series of prompt jumps at every time step, in- 
TABLE XIV

Percent Errors for a Delayed Critical Ramp.

\begin{tabular}{|c|c|c|c|}
\hline $\mathrm{h}$ (sec) & Core & Linear L $\phi, F \phi$ & Linear $\phi$ \\
\hline 0.10 & Left & 0.0181 & 4.31 \\
& Middle & 0.0260 & 6.93 \\
& Right & 0.0343 & 10.63 \\
0.05 & Left & 0.00507 & 1.42 \\
& Middle & 0.00730 & 2.28 \\
& Right & 0.00960 & 3.50 \\
& Left & $<0.001$ & 0.267 \\
0.02 & Middle & $<0.001$ & 0.430 \\
& Right & 0.00124 & 0.660 \\
\hline
\end{tabular}

TABLE XV

Ratios of Relative Error and Time Step Size.

\begin{tabular}{|l|c|c|c|}
\hline $\mathrm{h}$ (sec) & Left Core & Middle Core & Right Core \\
\hline 0.020 & 1.82 & 2.92 & 4.47 \\
0.010 & 1.89 & 3.04 & 4.67 \\
0.005 & 1.92 & 3.09 & 4.73 \\
0.0025 & 1.92 & 3.08 & 4.72 \\
\hline
\end{tabular}


stead of behaving smoothly as it should, and this in turn introduces an $O(h)$ error as a qualitative graphical analysis would show. Thus, one concludes that the analysis of sec. (2.4) is relevant only for $h$ extremely small. Table XV shows the ratio of the relative error and the time step size for the fast flux at the middle points of the three cores of the fast reactor, at $t=0.5 \mathrm{sec}$, for several values of $h$. The conclusion is immediate that the errors are indeed $0(h)$, for the time step sizes under consideration. In these calculations we used Method 1 with point kinetics thetas.

From the above results we infer that, for the point kinetics problem, the modified Crank-Nicholson method is probably also more accurate than the method of Chap. 2, for the case of a ramp in the range below prompt-critical except, perhaps, for slow ramps. This is because the latter method uses constant average parameters during a time step. Calculations were also done for a limited ramp in the thermal reactor, reaching into the prompt-supercritical range. Because of the small time steps needed to achieve a significant accuracy, Methods 1 and 2 essentially coincide. The ramp section was thus effectively treated by the modified Crank-Nicholson method, so there is nothing new here to compare results with. In the constant parameter section, improvement in accuracy by use of point kinetics thetas is comparable with the results shown in Case 3, as would be expected. 
We repeated some of the calculations mentioned before, using a constant weight function, instead of the adjoint flux for the initial state, to obtain the point kinetics parameters to find the thetas. The results for the cases explored did not differ greatly from the corresponding results using adjoint weighting. We found that while overall accuracy is somewhat better for adjoint weighting in some instances, this was not a systematic trend. In addition, we observed that the oscillations in the theta values tend to diminish with a constant weight function. Computation time savings by using a constant weight function might be appreciable, since it would eliminate all of the multiplications required in the scalar products needed to find the point kinetics parameters.

The use of point kinetics to calculate thetas for the ramp section in the cases analyzed did not produce good results. In general we found $\theta_{0} \simeq 0.5$, and deviations from this value were due more to the numerical procedure adopted than to a genuine deviation associated with the true point kinetics solution. Methods to improve accuracy of the numerical point kinetics calculation are straightforward but the computational effort spent in such a task is hardly justified. This prompted us to abandon point kinetics as a means of finding thetas, and to use $\theta_{0}=0.5$ under all ramp conditions. Good spectral coupling was observed for all transients. That is, the ratios of fast to slow fluxes for all points in both the thermal and the fast reactors deviated at most a 
few percent from steady-state values. This amount is quite small when compared with the large asymmetry factors encountered in the transients analyzed. This fact confirms our contention, made in Sec. (3.4), that the energy coupling is much stronger than spatial coupling and justifies our use of only one theta for all neutron energy groups, especially since only one theta is used for all spatial points.

Finally, we made special modifications in the computer code written to perform the calculations, in order to allow a comparison between the computation time using point kinetics thetas with that using predetermined thetas. It was found that, with adjoint weighting, use of point kinetics increased the computation time during a time step by about $50 \%$ for the fast reactor $(I=1)$ and by about $75 \%$ for the thermal reactor $(I=6)$. These numbers reflect both the time spent to do the point kinetics calculations (a function of I) and the time spent to obtain the point kinetics parameters, which depends not only on $I$ but also on $G$ and $K$ (see Chap. 3 for notation). This extra time would be somewhat reduced by use of a constant weight function.

For higher dimensions and more neutron energy groups, the fraction of time spent in point kinetics related computations may decrease, since the time spent for the matrix inversions required by implicit methods, will probably increase at a faster rate than the time spent for computing point kinetics thetas. 
Chapter 5

CONCLUSIONS AND RECOMMENDATIONS

\subsection{Conclusions for Point Kinetics}

As the analysis of the point kinetics results in Sec. (4.2) indicates, a great improvement is achieved in the simple Crank-Nicholson method by the addition of terms to treat the eigenvalues $\omega_{0}$ and/or $\omega_{I}$ explicitly, at least in cases of constant reactivity. This procedure allows much larger time steps to be taken while maintaining errors acceptably low, and yields a far superior accuracy for a given time step size.

The Padé $(2,0)$ method is also definitely superior to the Crank-Nicholson method, for constant reactivity, and does fairly well without an explicit treatment of any eigenvalue in many practical cases. The $\omega_{I}$ term is not essentially needed for the Padé $(2,0)$ approximation and the $\omega_{0}$ term need be included only for cases near or above prompt-criticality. The simple Padé $(2,0)$ approximation does not exhibit the oscillatory behavior sometimes observed in the Crank-Nicholson method.

In most instances, the method based on the Padé $(1,1)$ approximation produces better results than when the Padé $(2,0)$ expression is used in the present method. However, halving the time step size $h$ for the latter method will make it more accurate than the first method with time step $h$. 
Since the Padé $(1,1)$ method will, in many cases, require one more eigenvalue $\left(\omega_{I}\right)$ treated explicitly, and since this addition will normally more than double the computation time per step, we conclude that, usually, the method based on the Pade $(2,0)$ approximation will be faster, for the same accuracy.

For cases in which the reactivity changes with time, addition of eigenvalue correction terms to the basic Padé approximations did not produce significant improvements in accuracy.

From the above, we can conclude that, for cases of practical interest, the Padé $(2,0)$ method with a criterion to decide whether the $\omega_{0}$ term should be treated explicitly, plus some strategy for the choice of the time step size will, in general, provide a very fast and accurate computational method for point kinetics calculations in general, when the inversion scheme developed in Sec. (2.3) is used. For cases in which a very precise treatment of the beginning of transients is desired, the Padé $(1,1)$ method with inclusion of the $\omega_{6}$ term is to be preferred, when $\omega_{6}$ has $a$. large magnitude. Although one could also include the $\omega_{6}$ term in the Padé $(2,0)$ method, the error for the previous method will be smaller for most cases, as can also be concluded from the underlying theory.

The final conclusion is that the general method presented in Chap. 2, including the inversion scheme developed, is particularly good for cases in which the reactivity can 
be represented by a series of steps and performs quite well for more general cases.

\subsection{Conclusions for Space-Dependent Kinetics}

As stated in Sec. (4.3) it is difficult to draw general conclusions about which of the methods used in the calculations is the best, because of the lack of a general trend. On the other hand, some specific conclusions have been reached as summarized below.

The theta method based on point kinetics, for either Method 1 or Method 2, provides only a marginal improvement, in most cases, over these same methods with properly chosen fixed thetas. In some problems, the calculated thetas exhibit an oscillatory behavior that degrades the accuracy. Such behavior was even observed in a case of constant flux shape.

The point kinetics thetas did improve the accuracy (with respect to suitably chosen fixed thetas) in certain problems, when reactor parameters were constant for a period of time, but that improvement was, in many cases, restricted to the beginning of such period. Also, as the time step size decreased, point kinetics thetas tended to make results less accurate as compared to those obtained from properly chosen fixed thetas.

Methods 1 and 2 produce results of comparable accuracy. In subcritical problems, Method 1 was always better, the 
converse being true for delayed supercritical transients. The two Methods ( 1 and 2 ) essentially agree for promptsupercritical transients, because of the necessarily small time steps one has to take in such cases.

A case in which there was a significant improvement in accuracy with the point kinetics thetas was that of a prompt-supercritical step. This is acase in which one mode is strongly dominant. We feel, however, that one might try to find the asymptotic period in such cases by some simpler method and then use it to determine asymptotic thetas, to get a similar improvement without the need for the point kinetics calculations.

One definite conclusion reached was that use of $\theta_{0}=1$ for only the first one or two time steps of the calculation yields much better results than using $\theta_{0}=1$ for all time steps. This conclusion applies to cases in which using $\theta_{0}=0.5$ all the way would produce large errors and oscillations, as in cases below prompt-criticality where a constant reactivity section of a transient is preceded by a jump (step) or steep ramp. The decision of how steep the ramp is will depend on the problem at hand, as will the choice of how many steps to take with $\theta_{0}=1$. Another interesting conclusion, valid at least for the range below prompt-criticality, is that the approximations of linear $L \Phi$ and $F \Phi$ produce far better results than those obtained using the real $L(t)$ and $F(t)$ and then approximating 
$\Phi(t)$ by a straight line. This result suggests that higher order spline approximations for the time variable might become more accurate if applied to $L \Phi$ and $F \Phi$ rather than to $\Phi$ alone.

For reactors below prompt-critical at least, use of the constant parameter approximation, in which varying quantities are replaced by their average during the time step, also fared much worse when compared to the linear L $\phi$ and F $\phi$ approximations. The constant parameter approximation turns out to be $O(h)$ in the prompt-subcritical range, instead of $O\left(h^{2}\right)$, and becomes $O\left(h^{2}\right)$ only for very small values of $h$. The computation of the thetas using point kinetics took about $50 \%$ to $75 \%$ longer during the time step calculations, when adjoint weighting was used. Use of a constant instead of the adjoint flux as a weight function was also explored and found to yield results of comparable accuracy, with some savings in computation time.

Therefore, it is our final conclusion that the use of methods based on point kinetics calculations to find better integration parameters $\theta$ for the theta method are not. worth the computational effort required.

\subsection{Recommendations for Further Research}

The time step size that both the theta method and our point kinetics method can take, with satisfactory accuracy, is already quite large, in comparison with some other methods. While it can be argued that extending this time step 
much longer might produce the unwanted effect of having the flux grow so much that the feedback effects will be imprecisely accounted for, it must not be forgotten that, usually, methods that can take larger time steps mantaining a certain accuracy; have smallex errors for a given time step. Having smaller errors is, of course, a desirable feature. In this vein, we make the recommendations that follow. For point kinetics, one direction in which some additional study could be done is that of finding a better treatment for the case of reactivity varying in a general manner. A more difficult task would be to include feedback directly into the solution method. It appears to us, however, that an extension of the general method of chap. 2 in these directions does not seem to offer much promise.

The theta method, as considered in this thesis, is based on the idea of finding space-independent $\theta$-parameters that would improve the accuracy of the time integration of the space-dependent equations. It is our recommendation that the theta method as such should not be pursued further, as there appears to be little room for improvement over simpler choices of thetas like 0.5 and 1 .

A possible extension of the theta method, that does deserve some exploring, is that of finding space (and energy) dependent thetas that could improve the accuracy, along the lines described in Ref. (47) for the heat conduction equation. There, a $\theta$-matrix is found and incorporated direct- 
1y in the differencing scheme to produce a difference equation of higher accuracy. If this could be done for the reactor case without too much extra computational effort it would certainly be worthwhile. 


\section{REFERENCES}

1. A. F. HENRY and A. V. VOTA, "WIGL-2 - A Program for the Solution of the One-Dimensional, Two-Group Space-Time Diffusion Equations Accounting for Temperature, Xenon, and Control Feedback", WAPD-TM-532, Bettis Atomic Power Laboratory (1965).

2. J. B. YASINSKY, M. NATELSON and L. A. HAGEMAN, "TWIGL A Program to Solve the Two-Dimensional, Two-Group SpaceTime Neutron Diffusion Equations with Temperature Feedback", WAPD-TM-743, Bettis Atomic Power Laboratory (1968).

3. A. F. HENRY, Nucl. Sci. Eng., 3, 52 (1958).

4. E. P. GYFTOPOULOS, "General Reactor Dynamics", in the Technology of Nuclear Reactor Safety, Vol. 1, Chap. 3, T. J. THOMPSON and J. G. BECKERLEY, Eds.; The MIT Press, Cambridge, Massachusetts (1964).

5. Z. AKCASU, G. S. LELLOUCHE and L. M. SHOTKIN, Mathematical Methods in Nuclear Reactor Dynamics, Academic Press, New York and London (1971).

6. K. O. OTT and D. A. MENELEY, Nucl. Sci. Eng., 36, 402 (1969).

7. S. KAPLAN, "Synthesis Methods in Reactor Analysis", in Advances in Nuclear Science and Technology, Vol. 3, p. 233, P. GREEBLER and E. J. HENLEY, Eds., Academic Press, New York and London (1966).

8. T. A. PORSCHING, SIAM J. Appl. Math, 16, 301 (1968).

9. J. LEWINS, Nucl. Sci. Eng., 7, 122 (1960).

10. J. C. VIGIL, Nucl. Sci. Eng., 29, 392 (1967).

11. T. AUERBACH, J. MENNIG and G. SARLOS," The Application of Lie Series to Time Dependent Reactor Theory", in Proc. Brookhaven Conf. on Ind. Needs and Acad. Research 
in Reactor Kinetics, BNL-50117, p. 306, Brookhaven National Laboratory (1968).

12. G. R. KEEPIN and C. W. Cox, Nucl. Sci. Eng., 8, 670 (1960) :

13. W. R. RHYNE and A. C. LAPSLEY, Nucl. Sci. Eng, 40,91 (1970).

14. J. J. KAGANOVE, "Numerical Solution of the One-Group, Space-Independent Reactor Kinetics Equations for Neutron Density Given the Excess Reactivity", ANL-6132, Argonne National Laboratory (1960).

15. E. R. COHEN, "Some Topics in Reactor Kinetics", in Proc. 2nd U. N. Intern. Conf. Peaceful Uses At. Energy, 11, 302 (1958).

16. K. F. HANSEN, B. V. KOEN and W. W. LITTLE, Jr., Nucl. Sci. Eng., 22, 51 (1965).

17. C. M. KANG, Trans. Am. Nuc1. Soc., 14, 201 (1971).

18. T. A. PORSCHING, Nucl. Sci. Eng., 25, 183 (1966).

19. K. F. HANSEN, E. R. KLEBAN and I. J. CHANG, Trans. Am. Nucl. Soc., 12, 617 (1969).

20. M. IZUMI and T. NODA, Nucl. Sci. Eng., 4l, 299 (1970).

21. A. F. HENRY, Personal Communication (January 1970).

22. M. CLARK, Jr, and K. F. HANSEN, Numerical Methods of Reactor Analysis, Sec. 1.12, Academic Press, New York and London (1964).

23. E. ISAACSON and H. B. KELLER, Analysis of Numerical Methods, Sec. 1, John Wiley \& Sons, Inc., New York, London and Sidney (1966).

24. R. S. VARGA, Matrix Iterative Analysis, Sec. 8.3, Prentice-Hall, Inc., Englewood Cliffs, New Jersey (1962).

25. M. CLARK, Jr., and K. F. HANSEN, Op. Cit., Sec. 4.5. 
26. K. F. HANSEN, "A Comparative Review of Two-Dimensional Kinetics Methods", GA-8169, General Atomic Division of General Dynamics (1967).

27. C. G. CHEZEM and W. H. KOEHLER, Eds., "Coupled Reactor Kinetics", Proc. Nat. Topical Meeting Am. Nucl. Soc., Texas A\&M Press, College Station, Texas (1967).

28. S. KAPLAN, Nuc1. Sci. Eng., 9, 357 (1961).

29. W. M. STACEY, Jr., Modal Approximations, The MIT Press, Cambridge, Massachusetts (1967).

30. S. KAPLAN, O. J. MARLOWE, and J. BEWICK, Nucl. Sci. Eng., 18,163 (1964).

31. W. M. STACEY, Jr., Nucl. Sci. Eng., 34,45 (1968).

32. J. B. ANDREWS, II, and K. F. HANSEN, Nucl. Sci. Eng.' 31, 304, (1968).

33. W. T. MCCORMICK, Jr., and K. F. HANSEN, "Numerical Solution of the Two-Dimensional Time-Dependent Multigroup Equations", in Proc. Conf. on Effective Use of Computers in Nucl. Ind., CONF-690401, p. 76, USAEC (1969).

34. W. H. REED and K. F. HANSEN, Nucl. Sci. Eng., 41, 431 (1970).

35. A. L. WIGHT, K. F. HANSEN and D. R. FERGUSON, Nucl Sci. Eng., 44, 239 (1971).

36. D. R. FERGUSON and K. F. HANSEN, "Solution of the SpaceDependent Reactor Kinetics Equations in Three Dimensions", MIT-3903-4 (MITNE-132), Ph. D. Thesis, Department of Nuclear Engineering, Massachusetts Institute of Technology (1971).

37. T. A. PORSCHING and A. F. HENRY, "Some Numerical Methods for Problems in Reactor Kinetics"," in Numerical Solution of Field Problems in Continuum Physics, SIAM-AMS Proc., Vol. 2, p. 95, G. BIRKHOFF and R. S. VARGA, Eds., American Mathematical Society, Providence, R.I. (1970). 
38. J. B. YASINSKY and L. A. HAGEMAN, "On the Solution of the Time-Dependent Group Diffusion Equations by an Implicit Time Differenced Iterative Method", in Proc. Conf. on Effective use of Computers in Nucl. Ind., CONF690401, p. 55 , USAEC (1969).

39. W. M. STACEY, Jr., Space-Time Nuclear Reactor Kinetics, Chaps. 1-3, Academic Press, New York and London (1969).

40. R. D. RICHTMYER and K. W. MORTON, Difference Methods for Initial-Value Problems, 2nd. Ed., Chap. 3, Interscience Publishers (Wiley), New York, London and Sydney (1967).

41. L. A. HAGEMAN and J. B. YASINSKY, Nucl. Sci. Eng., 38, 8 (1969).

42. R. S. VARGA, Op. Cit., Sec. 1.5 .

43. R. S. VARGA, Op. Cit., Sec. 8.2.

44. E. I. WACHSPRESS, Iterative Solution of Elliptic Systems, Sec. 1.3, Prentice-Hall, Inc., Englewood Cliffs, N.J. (1966).

45. W. M. STACEY, Jr., to be published.

46. J. B. YASINSKY, Nucl. Sci. Eng., 32, 425 (1968).

47. W. M. STACEY, Jr., and C. H. ADAMS, Trans. Am. Nucl. Soc., 10, 251 (1967). 


\section{Appendix A \\ CALCULATION OF THE EIGENVALUES OF LARGE MAGNITUDE}

In what follows, we present a procedure to find the values of one or both of the two extreme eigenvalues of the point kinetics matrix $A$, which are the algebraically largest and smallest roots of the inhour formula, Eq. (2.3).

The calculation of $\omega_{0}$ or $\omega_{I}$ can be done by table lookup and interpolation, by using the Newton-Raphson method, or by a combination of both schemes. In this last method, a first guess is obtained from a table lookup and the Newton-Raphson method is used to improve upon the initial guess to any desired degree of accuracy.

The Newton-Raphson method can be shown to converge in the following cases:

a) For $\omega_{0}$, with some initial guess $\omega_{0}^{(0)}$ such that

$$
-\lambda_{1}<\omega_{0}^{(0)}<\omega_{0}
$$

b) For $\omega_{I}$ ' with some initial guess $\omega_{I}^{(0)}$ such that

$$
\omega_{I}<\omega_{I}^{(0)}<-\lambda_{I}
$$

It can also be shown that $\omega_{I}<\alpha<\omega_{0}$ and that, if $\alpha$ has a large enough magnitude, it is already a good approximation for one of the roots and can, thus, be used as a good first guess for that root. 
The Newton-Raphson expression is derived from Eq. (2.3) and is given by

$$
\left.\omega^{(j+1)}=\frac{\rho-\sum_{i=1}^{I} \beta_{i}\left(\frac{\omega}{\lambda_{i}+\omega}(j)\right.}{\Lambda+\sum_{i=I}^{I} \beta_{i} \lambda_{i}\left(\frac{1}{\lambda_{i}+\omega}(j)\right.}\right)^{2}
$$

In practice, with reasonable first guesses, two to five iterations are enough to achieve an accuracy of one part in $10^{5}$. In order to appreciate the amount of work involved in the above calculations we estimated the computer time necessary to do one iteration, which, by efficient programming, can be reduced to $3 I$ additions and subtractions, 4 I multiplications and $(I+1)$ divisions, for $I$ precursor groups. If we take $I=6$ and assume $t_{ \pm}=2 \mu \mathrm{sec}, t_{x}=4 \mu \mathrm{sec}$ and $t_{\div}=$ 7 usec (approximately the IBM $360 / 65$ times) one would spend about 180 usec per iteration which, for comparison, is about the time needed to calculate two exponentials in single precision (in the IBM $360 / 65$ ).

We can see, therefore, that the amount of work spent in the calculation of the desired roots is not unduly large, particularly in view of the relatively large time step sizes one will be able to take with the present method. 


\section{Appendix B \\ CALCULATION OF THE PARAMETERS THETA}

In this appendix we describe the procedure used to find the thetas for use in Eq. (3.10) when based on a point kinetics calculation. The notation adopted in Chap. 2 is used throughout except when otherwise noted.

The case of constant parameters is dealt with by direct use of the methods of Chap. 2. For Method 1 we write Eq. $(3.18)$ in the form

$$
\frac{1}{h}\left(\underline{\Psi}_{1}-\underline{\Psi}_{0}\right)=A\left[\theta \underline{\Psi}_{1}+(I-\theta) \underline{\Psi}_{0}\right]
$$

where $\theta=\operatorname{diag}\left[\theta_{0} \theta_{1} \theta_{2} \cdots \theta_{I}\right]$. From this and Eq. (2.2) without source we get

$$
\left[(h A)^{-1}\left(e^{h A}-I\right)-I\right] \Psi_{0}=\theta\left[e^{h A}-I\right] \Psi_{0}
$$

which, as we shall see, is defined even if A does not have an inverse. Using Eq. $(2.8)$ with $f(h A)$ given by the Padé $(1,1)$ approximation, $f_{1}(h A)$, we get

$$
\begin{aligned}
{\left[\frac{1}{2} g(h A)+\right.} & \left.\sum_{k}^{1}\left[\frac{e^{h \omega_{k}}-f\left(h \omega_{k}\right)}{h \omega_{k}}\right] \underline{u}_{k} \underline{v}_{k}^{T}\right] \underline{\Psi}_{0}= \\
& \theta\left[g(h A)+\sum_{k}^{1}\left\{e^{h \omega_{k}}-f\left(h \omega_{k}\right)\right\} \underline{u}_{k} \underline{v}_{k}^{T}\right] \underline{\Psi}_{0}
\end{aligned}
$$

with $g(h A)=\left[I-\frac{1}{2} h A\right]^{-1} h A$, from which $\theta$ can be calculated. Note that, if no terms are included in $\Sigma^{\prime}, \theta=\frac{1}{2} \mathrm{I}$. 
If the Padé $(2,0)$ approximation, $f_{2}(h A)$, is used, there will, in general, be one less term in $\Sigma^{\prime}$. But the computation of the expressions that replace $g(h A)$ will be more complicated. Since the error is smaller for the Padé $(1,1)$ approximation, we decided to use it here.

We adopt the above procedure (i.e. Eq. (2)) to find the thetas since it is computationally simpler, and since it eliminates much of the error arising from the fact that Eq. (3.18) sometimes forms a quasi-singular system for the thetas. Equation (2) mathematically cancels the singularities due to small $h$ or to the fact that $A$ is singular when $\rho=0$.

For the case of constant parameters, Eq. (1) can also be intepreted as representing

$$
\frac{1}{\bar{h}} \int_{0}^{h} \underline{\Psi}(t+s) d s-\underline{\Psi}_{0}=\theta\left(\underline{\Psi}_{1}-\underline{\Psi}_{0}\right)
$$

as can readily be: verified by use of Eq. (2.2).

For Method 2 we determine $\theta_{0}$ and $\theta_{i}^{\prime}$ directly, rather than finding $\theta(s)$ and then using Eq. (3.14). When the definition of $\theta(s)$ in Eq. (3.19) and the expression for $\theta_{0}$ in Eq. (3.14) are applied, Eq. (3) shows that $\theta_{0}$ is the same for both Methods 1 and 2 , and can thus be calculated using part of Eq. (2).

Equation (3.14) for $\theta_{i}$ and $\tau_{i}$ leads to

$$
\theta_{i}^{\prime}=\frac{\frac{1}{\hbar} \int_{0}^{h} e^{-\lambda_{i}(h-s)} n(t+s) d s-\tau_{i} n_{0}}{n_{1}-n_{0}}=\frac{\frac{c_{i 1}-e^{-h \lambda_{i}} c_{i 0}}{h \mu_{i}}-\tau_{i} n_{0}}{n_{1}-n_{0}}
$$


where $\left(n_{1}-n_{0}\right)$ can be obtained in the process of calculating $\theta_{0}$

Note that we could try also to decrease the error in the numerator by writing it in the form

$$
\frac{1}{\mu_{i}}\left\{\left(\frac{c_{i 1}-c_{i 0}}{h}-\dot{c}_{i 0}\right)+\left(1-\tau_{i}\right) \dot{c}_{i 0}\right\}
$$

where the first part could be obtained by evaluating $\left[e^{h A}-I-h A\right] \Psi_{0}$ by a procedure similar to that used in Eq. (2). We have not implemented this procedure, however. 


\section{Appendix C \\ PROOF OF EQUIVALENCE OF NORMS}

The $I_{2}$ norm was defined in Sec. (3.6) as being

$$
\|\underline{u}\|=\left[\int_{V}|\underline{u}(r)|^{2} d v\right]^{1 / 2}=\left[\sum_{n} u_{n}^{2} w_{n}^{2}\right]^{1 / 2}
$$

the last form applying to spatially discrete functions or vectors (supervectors). The supervector index $\mathrm{n}$ depends both on the spatial index $\mathrm{k}$ and on the position index in $\underline{u}\left(r_{k}\right)$. Here, $w_{n}^{2}$ are integration weights, proportional to $\Delta \mathbf{r}$ and related to the interpolation procedure chosen to make the discrete functions continuous.

The Euclidean norm of a vector (or supervector) is simply

$$
\|\underline{v}\| \cdot=\left[\underline{v}^{T} \underline{v}\right]^{1 / 2}=\left[\sum_{n} v_{n}^{2}\right]^{1 / 2}
$$

so that, by defining $\Omega=\operatorname{diag}\left[w_{n}\right]$, we can write the relation between the $\mathrm{L}_{2}$ norm and the Euclidean norm as

$$
\|\underline{u}\|=\|\Omega \underline{u}\| \cdot
$$

Now, for a matrix $\mathrm{H}$ we have, by definition,

$$
\begin{gathered}
\|\mathrm{H}\|=\max _{\|\underline{\mathrm{u}}\|=1}\|\underline{\mathrm{u}}\|=\max _{\|\Omega \underline{u}\|^{\prime}=1}\|\Omega \mathrm{H} \underline{\underline{u}}\|^{\prime}= \\
=\max _{\|\underline{x}\|^{\prime}=1}\left\|\Omega \mathrm{H} \Omega^{-1} \underline{\underline{x}}\right\|^{\prime}=\left\|\Omega \mathrm{H} \Omega^{-1}\right\|^{\prime}
\end{gathered}
$$

where we made $\Omega \underline{u}=\underline{x}$ and used the definition of the Euclidean 
norm of a matrix ${ }^{(23)}$

In general

$$
\|\mathrm{H}\|=\left\|\Omega \mathrm{H} \Omega^{-1}\right\|^{\prime} \leq\|\Omega\|^{\prime}\|\mathrm{H}\|^{\prime}\left\|\Omega^{-1}\right\|^{\prime}=\frac{\mathrm{w}_{\max }}{\mathrm{w}_{\min }}\|\mathrm{H}\| \cdot
$$

and

$$
\|\mathrm{H}\|^{\prime}=\left\|\Omega^{-1} \Omega \mathrm{H} \Omega^{-1} \Omega\right\|^{\prime} \leq\left\|\Omega^{-1}\right\| \cdot\left\|\Omega \mathrm{H} \Omega^{-1}\right\| \cdot\|\Omega\|^{\prime}=\frac{\mathrm{w}_{\max }}{\mathrm{w}_{\min }}\|\mathrm{H}\|
$$

so that

$$
\mathbf{k}\|\mathrm{H}\|: \leq\|\mathrm{H}\| \leq \mathrm{K}\|\mathrm{H}\| \cdot
$$

where $\mathrm{k}=1 / \mathrm{k} \geq \mathrm{w}_{\max } / \mathrm{w}_{\min }$ is a fixed constant; if the spatial mesh size is shrunk to zero uniformly, that is, if $\Delta \mathrm{r}_{\max } / \Delta \mathrm{r}_{\min }$ is bounded by some constant. 


\section{Appendix D}

\section{INPUT CARDS FOR COMPUTER PROGRAMS}

\section{D.I Point Kinetics Programs}

Card 1: FORMAT(II0,4DI0.0). For the method based on

$$
\text { Padé }(1,1) \text {. }
$$

FORMAT $(I 10,3 D 10.0)$. For the method based on Padé $(2,0)$.

NPG = number of neutron precursor groups, I.

HWS $(1)=$ HWS $=$ number such that, if $h \omega_{0}>$ HWS; the $\omega_{0}$ term is included in $\Sigma$ ', Eq. $(2.8)$.

HWS (2) = number such that, if $h \omega_{I}<\min \left(\operatorname{HWS}(2),-1.2 \mathrm{~h} \lambda_{I}\right.$ ), the $\omega_{I}$ term is included in $\Sigma 1$, Eq. $(2.8)$. Not used with Pạdé $(2,0)$.

OMTOL = relative error for the computation of $\omega_{0}$ and $\omega_{I}$. LAMBDA = neutron generation time, $\Lambda$.

Card 2: FORMAT (6D10.0)

$(\operatorname{BETA}(I), I=1, N P G)=$ delayed neutron fractions, $\beta_{i} \cdot$

Card 3: FORMAT (6DI0.0)

$(\operatorname{LAM}(I), I=I, N P G)=$ decay constants, $\lambda_{i} \cdot$

Card 4: FORMAT(II0,4Dl0,0). One card for each time zone.

NPRINT = solution printed every NPRINT steps.

$\mathrm{H} \quad=$ time step size, $\mathrm{h}$. 
Tz = end time for the time zone.

RHO $\varnothing=$ initial value of the reactivity for the time zone, $\rho_{0}$.

DRHO = derivative of the reactivity with respect to time for the time zone, $\dot{\rho}_{0}$.

Observations: (a) $\rho(t)=\rho_{0}+\dot{\rho}_{0} t$.

(b) the programs treat only problems without external source, in their present version.

(c) to run several cases the whole set of cards described before ( 1 to 4 ) is repeated as many times as there are cases, with a blank card separating the set of cards for consecutive cases.

\section{2 One-Dimensional Space-Kinetics Program}

Card 1: FORMAT (5I5)

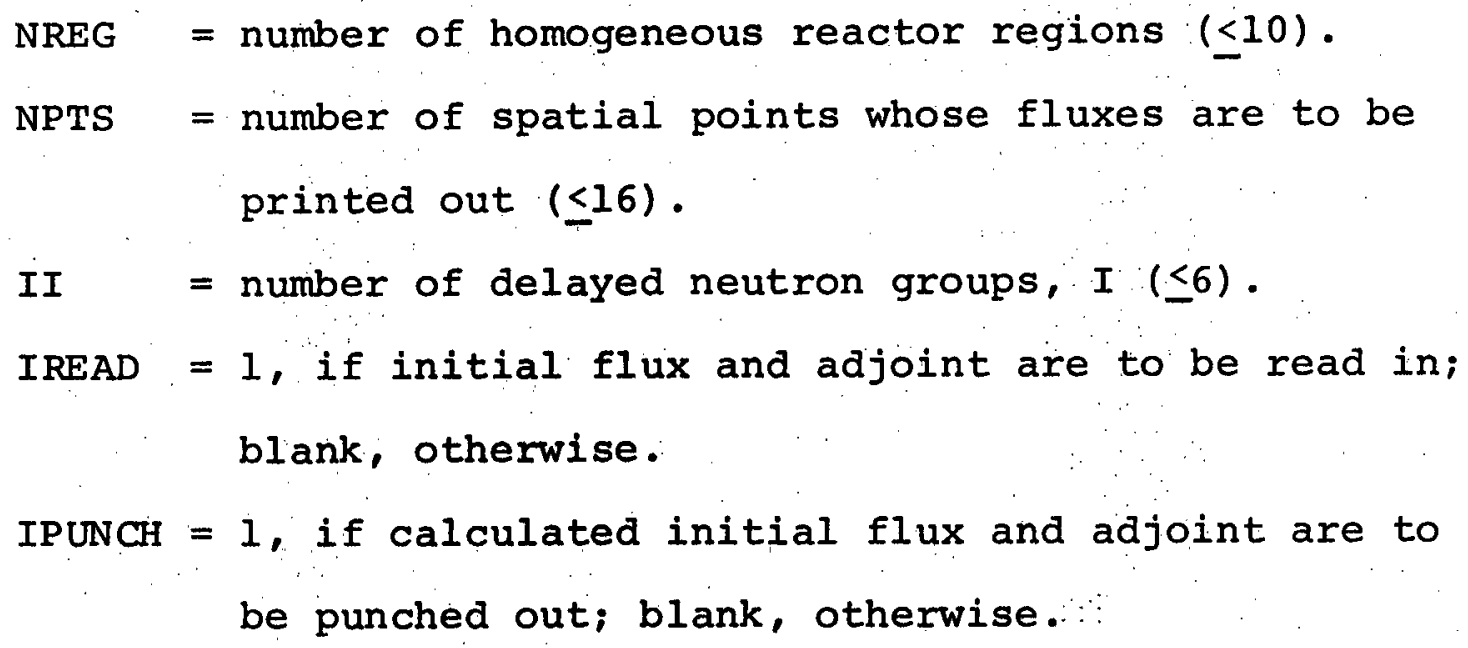


Card 2: FORMAT (16I5)

$(\mathrm{KPRINT}(\mathrm{N}), \mathrm{N}=1, \mathrm{NPTS})=$ spatial mesh point numbers of points whose fluxes will be printed out.

Card 3: FORMAT (2D10.0)

CRITOL = relative errors for eigenvalue $\left(k_{\text {eff }}\right)$ and for pointwise flux and adjoint.

OVER = overrelaxation parameter, between 1 and 2 .

Card $4:$ FORMAT $(4 \mathrm{D} 10.0)$

$(V(G), G=1,2):=$ group velocities; $v_{g^{*}}$

$(C H I O(G), G=1,2)=$ prompt neutron spectrum, $x_{0 g}$.

Card 5: FORMAT (6Dl0.0)

$(\operatorname{BETA}(I), I=1, I I)=$ delayed neutron fractions, $\beta_{i}$.

Card 6: FORMAT (6D10.0)

$(\operatorname{IAM}(I), I=1, I I)=$ decay constants, $\lambda_{i}$.

Card 7: FORMAT(6D10.0). One card for each neutron energy group.

$(\operatorname{CHII}(G, I), I=I, I I)=$ delayed neutron energy spectrum $X_{i g}{ }^{\circ}$

Card 8: A set of two cards for each reactor region.

Card 8-A: FORMAT (8DI0.0)
$(D(G), G=1,2)$
= diffusion constants, $\mathrm{D}_{\mathrm{g}}$.
$\left(\left(\operatorname{SIG}\left(G, G^{\prime}\right), G^{\prime}=1,2\right), G=1,2\right)=$ cross section matrix, $\sum_{g g^{\prime}}^{\prime}=$ 
$=\Sigma_{\mathrm{Tg}} \delta_{\text {gg }}-\Sigma_{\text {sgg }} \cdot$

$(\operatorname{SFN}(G), G=1,2)$

$=$ neutron production cross sections, $\nu \Sigma_{f g} \cdot$

Card 8-B: FORMAT (2D10.0)

$\mathrm{x}=$ width of the region.

$\mathrm{KINT}=$ number of mesh intervals in the region.

Card 9: FORMAT(4D20.0). One card for each interior mesh point, if IREAD $=1$.

$(\operatorname{FLUX}(G, K), G=1,2)=$ initial flux, $\emptyset_{\mathrm{gk}}$.

$(\operatorname{ADJ}(G, K), G=1,2)=$ initial adjoint, $\emptyset_{g k}^{*}$.

Card 10: FORMAT (I5)

NTZ = number of time zones.

Card 11: A set of (NREG+1) cards for each time zone.

Card 11-A: FORMAT(I5,D10.0)

IRAMP $=0$ if step, $I$ if ramp.

$\mathrm{TZ}:=$ end time for the time zone.

Card 11-B : FORMAT(4D10.0). One card for each reactor region.

(DSA $(G), G=1,2)=\Delta\left(\Sigma_{a g}\right)$ if step, or $d\left(\Sigma_{a g}\right) / d t$, if ramp. $(D S F N(G), G=1,2)=\Delta\left(\nu \Sigma_{f g}\right)$ if step, or $d\left(\nu \Sigma_{f g}\right) / d t$, if ramp.

Card 12: FORMAT(2D10,0,I10). One for each time zone.

H = time step size. 


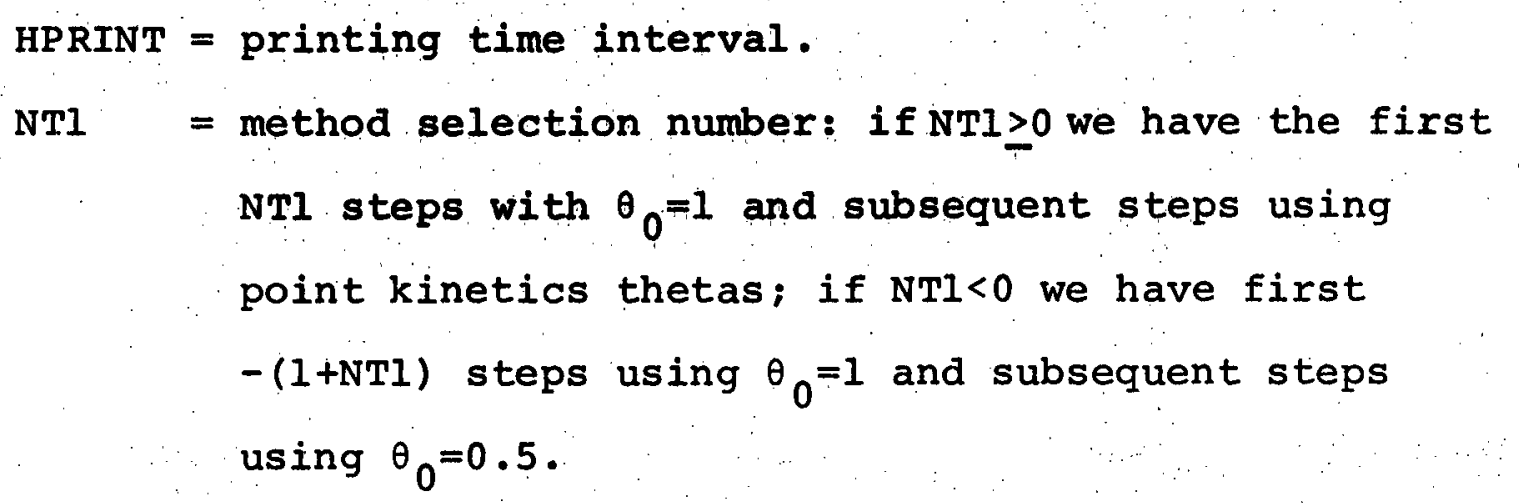

Observations: (a) $\Sigma\left(0^{+}\right)=\Sigma\left(0^{-}\right)+\Delta \Sigma$, if step and $\Sigma(t)=\Sigma(0)+t \dot{\Sigma}$,

if ramp. For constant parameter zone without initial jump it is more efficient to take step with $\Delta \Sigma=0$ instead of ramp with $\dot{\Sigma}=0$.

(b) the maximum number of inner mesh points is 100 .

(c) the program treats only two-group slab reactors with zero flux boundary conditions at both boundaries.

(d) to run the same problem with different $H$, HPRINT, or NTI repeat card set 12 (NTZ cards) as many times as desired, separating consecutive sets by a blank card.

(e) to run different transients for the same reactor, repeat card sets 10 to 12 as many times as desired, separating consecutive transients by a card with 1 punched in the first column.

(f) to run different transients in different 
reactors, repeat all cards sets from 1 to 12, as many times as desired, separating consecutive cases by a card with 2 punched in the first column.

(g) any combination of possibilities described in items (d), (e), and (f) is permitted.

(h) logical unit 8 is used for input/output so that a control card for FTO8F001 has to be included. 


\section{Appendix $\mathbf{E}$ \\ LISTINGS OF COMPUTER PROGRAMS \\ (only in first six copies)}

The following pages contain the source listings of the computer programs enumerated below, which were used to do the calculations in this thesis.

\section{E.1 Point Kinetics Programs}

E.1.1 Method Based on the Padé $(2,0)$ Approximation
a) MAIN PROGRAM
b) FUNCTION REACT
c) SUBROUTINE ROOT

E.1.2 Method Based on the Padé $(1,1)$ Approximation
a) MAIN PROGRAM
b) FUNCTION SUM
c) SUBROUTINE ROOT
d) SUBROUTINE NEWRAP

\section{E.2 One-Dimensional Space-Kinetics Program}
a) MAIN PROGRAM
b) SUBROUTINE INPUT
c) SUBROUTINE PREP
d) SUBROUTINE CRITIC
e) SUBROUTINE INVERT
f) SUBROUTINE PQP
g) SUBROUTINE THETA (for Method 1) SUBROUTINE THETA (for Method 2)
h) SUBROUTINE POINTK (for Method 1) SUBROUTINE POINTK (for Method 2)
i) SUBROUTINE ROOT
j) SUBROUTINE SOURCE 